\title{
Acquired resistance to PD-L1 inhibition is associated with an enhanced type I IFN-stimulated secretory program in tumor cells
}

\author{
Yuhao Shi ${ }^{1}$, Melissa Dolan ${ }^{1}$, Michalis Mastri², James W. Hill ${ }^{3}$, Adam Dommer ${ }^{2}$, \\ Sebastien Benzekry ${ }^{4}$, Kevin Eng ${ }^{2,5}$, and John M.L. Ebos ${ }^{1,2,6}$
}

1 Department of Experimental Therapeutics, Roswell Park Comprehensive Cancer Center, Buffalo, NY, 14263. USA

2 Department of Cancer Genetics and Genomics, Roswell Park Comprehensive Cancer Center, Buffalo, NY, 14263. USA

3 Jacobs School of Medicine and Biomedical Sciences, SUNY at Buffalo

4 COMPutational pharmacology and clinical Oncology, Inria Sophia - Antipolis, Centre de Recherches en Cancérologie de Marseille, Inserm U1068, CNRS UMR7258, Institut PaoliCalmettes , Faculté de Pharmacie, Aix-Marseille University

5 Department of Biostatistics and Bioinformatics, Roswell Park Comprehensive Cancer Center, Buffalo, NY, 14263. USA

6 Department of Medicine, Roswell Park Comprehensive Cancer Center Buffalo, NY, 14263. USA

* Correspondence: John M.L. Ebos, Roswell Park Comprehensive Cancer

Center, Center for Genetics and Pharmacology, Elm and Carlton Streets, Buffalo, NY 14263-0001. Phone: 716-845-8233; Fax: 716-845-8232; E-mail:

John.Ebos@RoswellPark.org 


\begin{abstract}
Therapeutic inhibition of programmed cell death ligand (PD-L1) can reverse PD-1-mediated suppression of tumor-killing T-cells; however, many patients develop resistance. Acquired resistance may be derived from intracellular PD-L1 and interferon(IFN) signaling programs in the tumor that can have dual, sometimes opposing, influences on tumor immune responses. Here we show that PD-L1 inhibition induces a novel secretory program tightly controlled by IFN-signaling and specific to acquired, but not innate, resistance in tumors. A PD-L1 treatment-induced secretome (PTIS) was found to be enriched for several IFN-stimulated genes (ISGs) and then further enhanced by type I IFN stimulation (IFN $\alpha$ or IFN $\beta$ ) in multiple mouse tumor models. Chronic inhibition or gene knockout of tumor PD-L1 in vitro could elicit similar type I IFNenhanced secretory stimulation while resistant cells were able to suppress $\mathrm{T}$ cell activation and killing ex vivo. When reimplanted into mice, resistant tumors were more sensitive to IL-6 inhibition (a key PTIS component) and growth significantly reduced when type I IFN signaling was blocked. Together, these results show that prolonged PD-L1 inhibition can 'rewire' existing intracellular IFN:PD-L1 signaling crosstalk to drive secretory programs that help protect tumors from immune cell attack and represent a targetable vulnerability to overcome acquired resistance in patients.
\end{abstract}




\section{Introduction}

Cancer therapies can provoke unexpected (and often unwanted) cellular reactions that include the secretion of proteins such as growth factors and cytokines - many of which have been exploited as possible biomarkers of treatment effect or toxicity in patients ${ }^{1-3}$. Such therapyinduced secretomes (TIS) can also contribute to cancer progression, particularly in settings of acquired resistance where tumor cell populations adapt to treatments over prolonged periods ${ }^{3}$. For immune-checkpoint inhibitors (ICIs) that target the programmed cell death 1 (PD-1) pathway, early cytokine changes (e.g. IL6, IL8) in patients after treatment can correlate with initial responses ${ }^{4,5}$ or adverse events ${ }^{6}$, but whether tumor-specific secretory profile changes can be a cause (or consequence) of acquired resistance remains unclear ${ }^{3,7}$. This may be because blockade of programmed death ligand 1 (PD-L1) expressed on tumor cells is typically thought to inhibit growth primarily via tumor-extrinsic functions, namely, by restoring the cell-killing functions of cytotoxic T cells controlled by PD-1 signaling ${ }^{8,9}$. It is perhaps less appreciated that tumor-intrinsic intracellular functions of PD-L1 can also help tumors evade immune detection ${ }^{8}$. Indeed, PD-L1 has signaling crosstalk with multiple processes such as mTOR/AKT ${ }^{10,11}$, MAPK ${ }^{12}$, STAT3/Caspase $7{ }^{13}$, integrin $\beta 4^{14}$, MerTK ${ }^{15}$, BIM/BIK ${ }^{16}$ - all of which can protect tumor cells from T cell-mediated cytotoxicity ${ }^{17}$ (see ${ }^{18}$ for review).

In this regard, the effect of interferons (IFNs) on tumoral control of secretory programs following acquired resistance to PD-L1 inhibition may be of interest for several reasons ${ }^{3,19}$. First, Gato-Canas and colleagues recently showed that PD-L1 can signal via three conserved intracellular motifs and exert direct regulatory control of IFN-mediated cytotoxicity through inhibition of STAT3/Caspase $7^{13}$. Second, IFNs have been linked to multiple ICI treatment resistance mechanisms mostly via the induction of IFN-stimulated genes (ISGs) activated by type 
I $(\alpha / \beta)$ and type II $(\gamma)$ IFN subtypes. Currently the precise effect of IFNs on PD-L1 inhibitor efficacy remains enigmatic because they can, somewhat paradoxically, both protect and weaken immune defenses (often simultaneously) ${ }^{20,21}$. For instance, IFNs can boost antigen presentation (e.g. via beta-2-microglobulin and MHC-I expression) to improve PD-1 inhibitor responses ${ }^{22,23}$, while also suppressing immune cell attack via the induction of T-cell inhibitory ligands ${ }^{20,24}$, NOS2 25 , and SerpinB9 ${ }^{26}$, amongst many others ${ }^{27,28}$. Finally, IFNs also can regulate a range of cellular processes that involve additional cytokine production ${ }^{29,30}$ that, in turn, can have positive and negative effects on tumor progression. Currently it is unknown whether these IFN-controlled secretory programs are enhanced (or inhibited) in tumor cells in the context of acquired resistance, where persistent PD-L1 blockade may impact immune-protective processes.

Here we used treatment-sensitive mouse models to generate PD-L1 drug resistant (PDR) tumor cells to evaluate changes in secretory profiles. Using transcriptomic and proteomic analysis, we identified a PD-L1 treatment-induced secretome (PTIS) signature that was enriched for ISGs and could be validated in multiple datasets involving PD-L1 therapy. Type I IFN $\beta$ stimulation was found to potently enhance PTIS expression, even in tumor cells chronically exposed to drug in vitro, suggesting direct drug actions can alter secretory machinery controlled by PD-L1:IFN signaling crosstalk. PDR cells were also found to have enhanced immune protection from CD8+ T cell cytotoxicity and blockade of specificPTIS ISGs (such as IL-6) or IFN receptor that enhanced anti-tumor effects. Together, these results show that tumor-intrinsic immune protective secretory changes occur following acquired resistance to PD-L1 inhibition and identify a novel rationale for targeting type I IFNs to improve patient outcomes after treatment failure. 


\section{Results}

\section{Acquired resistance to PD-L1 inhibition increases secretory profiles enriched for type I IFN} regulated genes

To examine acquired resistance to PD-L1 inhibition, the PD-1 pathway inhibitor-sensitive murine breast tumor EMT6 cell line ${ }^{31-33}$ was implanted orthotopically in Balb/C mice and treated with aPD-L1 (clone 80) or IgG control antibody (Fig 1a; schematic shown). Following continuous treatment, a PD-L1 drug-resistant (PDR) cell variant (EMT6-PDR) was selected from mice with tumors that resumed growth after an initial significant delay (Fig 1b; circles shown). Transcriptome RNA-sequencing of EMT6-PDR and EMT-P (parental) tumor tissues revealed multiple genes to be up- or down-regulated (Fig 1c). Gene-set enrichment analysis (GSEA) showed EMT6-PDR tumors to be significantly enriched for genes associated with extracellular matrix, growth factor, and cytokine signaling pathways, several of which were secreted and IFNregulated (Fig 1d). Using the Gene Ontology (GO) database term GO:0005576 consisting of products outside or unattached to the cell ${ }^{19}$, secretory genes were found to increase in EMT6-PDR tumor transcripts and associate with inflammatory signaling, wound healing, and immune cell function/migration (Fig 1e). Since many of these processes also associate with IFN signaling ${ }^{30,34}$, we examined IFN-regulated genes using the Interferome database - a compilation of published in vitro and in vivo experimental datasets identifying transcriptomic and proteomic changes after IFN treatment ${ }^{35}$. Compared to P controls, EMT6-PDR tumors had several IFN-related genes up- and down-regulated (54\% and 63\%, respectively), with type I IFN gene upregulation the most common (22\% of total) (Fig 1f). Several IFN stimulated genes (ISGs) and type I IFNs were confirmed to be upregulated in EMT6-PDR cells using qRT-PCR analysis (Fig 1g). Next, we tested whether IFN gene enrichment could also be detected in tumors innately resistant to $\alpha$ PD-L1 treatment. To 
do this, we first implanted the PD-1 pathway inhibitor-insensitive murine kidney tumor RENCA cell line orthotopically into Balb/C mice ${ }^{36}$ and, following treatment with $\alpha \mathrm{PD}-\mathrm{L} 1$ or IgG antibody

(Fig 1h; schematic shown), selected a RENCA-PDR tumor cell variant that did not respond to treatment (Fig 1i; circles shown). EMT6-PDR and RENCA-PDR cells were then compared for relative enrichment of IFN signaling gene-sets found in several publications ${ }^{20,21,37-39}$ and in the Hallmark Molecular Signatures Database (MSigDB) ${ }^{40}$ (Table S1), with only EMT6-PDR cells showing positive enrichment (Fig 1j; RENCA-PDR negatively enriched). Next, a similar comparison was performed using an IFN $\gamma$-associated gene-set identified in durvolumab-treated non-small cell lung carcinoma (NSCLC) patient tumor biopsies ${ }^{41}$, with only EMT6-PDR cells showing positive enrichment (Fig 1k). Taken together, these results demonstrate that $\alpha \mathrm{PD}-\mathrm{L} 1$ treatmentcan induce ISG-related secretory gene changes in tumors that may be specific to acquired, but not innate, resistance settings.

\section{A PD-L1 treatment-induced secretome (PTIS) is enriched in PD-L1 treatment-sensitive}

\section{clinical and preclinical models}

To examine this further, we next tested whether IFN-enriched secretory effects found after acquired resistance were unique to tumor models reported to be initially sensitive to PD-L1 treatment. To do this, we developed a composite of secretory genes and proteins found to be increased in EMT6-PDR tumors based on RNAseq, qRT-PCR, cytokine array, andELISA analysis (Fig S1; see Methods). From this, we identified a $\alpha$ PD-L1 treatment-induced secretome (PTIS) signature consisting of 13 up-regulated molecules, with 12/13 representing IFN-regulated genes identified via the Interferome Database (Fig 2a). We next tested for PTIS signature enrichment in publicly available NCBI GEO and dbGAP whole tran scriptome datasets from published preclinical 
and clinical studies involving $\alpha$ PD-L1 treated tumors. In 5 preclinical studies examined, 3 were reported as $\alpha \mathrm{PD}-\mathrm{L} 1$ treatment-sensitive (Lan et ${ }^{31}{ }^{31}$; Sceneay et al ${ }^{42}$; Efremova et ${ }^{43}$ ) and 2 were $\alpha \mathrm{PD}-\mathrm{L} 1$ treatment-insensitive (Sceneay et al ${ }^{42}$ and RENCA-PDR tumor cells from this study) (See Methods for details). PTIS signature expression was found to increase in all $\alpha$ PD-L1 treatmentsensitive models as defined by average counts per million (CPM) levels, with 2 of 3 models demonstrating significant positive GSEA enrichment and 1 of 3 models showing significance by both CPM expression and GSEA enrichment(Fig 2b). Conversely, PTIS signature expression was decreased in $\alpha$ PD-L1 treatment-insensitive models (Fig 2c). In 2 clinical studies examined, tumor biopsies were taken from non-small cell lung carcinoma (NSCLC) (Gettinger et al. ${ }^{44}$ ) and merkel cell carcinoma (MCC) (Paulson et al. ${ }^{45}$ ) patients reported to be initially sensitive to $\alpha \mathrm{PD}-\mathrm{L} 1$ treatment (See Methods). In the NSCLC study, the PTIS signature expression was increased and significant positive GSEA enrichment found in bulk RNAseq data from patients who developed acquired resistance (Fig 2d). In MCC, single-cell RNAseq datasets also had increased PTIS signature expression in tumor, macrophage, and T-cell enriched cell compartments after clustered analysis (Fig 2e; See Methods). Notably, we generated a separate PTIS using only genes downregulated in EMT6-PDR cells (termed 'PTISDOwN'); however, similar dataset validations were not consistent suggesting the upregulated PTIS is more representative of acquired resistance (Fig S2a; See Supplemental Results). Together, these findings demonstrate that the IFN-enriched PTIS is increased in multiple preclinical and clinical tumors initially sensitive to $\alpha$ PD-L1 treatment, and occurs independent of cancer type.

\section{Type I IFN stimulation enhances PTIS expression in PDR cells}


Since many ISGs found in the PTIS can be regulated by type I IFNs $(\alpha, \beta)$ binding to IFN alpha receptor (IFNAR) ${ }^{46}$, we next examined whether IFN stimulation could impactPTIS after acquired resistance. To test this, EMT6-P and PDR cells were incubated with recombinant type I IFNs ( $\alpha$ or $\beta$ ) or type II IFN $(\gamma)$ for 48 hours (10ng/ml). Multiple ISGs (IL6, Nos2, Cxcl9, Rsad2) found to be upregulated in EMT6-PDR cells were further enhanced by IFN stimulation (Fig 3a; exception Cxc19). Notably, of the PTIS factors tested, IL6 expression increases were the most robust in EMT6-PDR cells before and after type I IFN stimulation (Fig 3b; heatmap summarizing relative expression shown). This stimulatory effect was confirmed in protein studies using conditioned media (CM), where IFN $\beta$ was found to be the strongest inducer of IL6 after resistance (Fig 3c). Notably, anti-proliferative effects of IFN treatment ${ }^{47}$ were not found to be consistently different amongst EMT6-P and PDR cells (Fig S3). To further examine whether IFN-enhanced PTIS is primarily regulated by type I IFN signaling, IFNAR1 was knocked down in EMT6-P and PDR cells (designated IFNAR1 ${ }^{\mathrm{KD}}$ ) via short hairpin RNA (Fig 3d). Results showed that increases in IL6 expression by IFN $\beta$-stimulation could be reversed in EMT6-PDR-IFNAR1 KD cells (Fig 3e). Finally, since tumoral PD-L1 intrinsic functions and expression have been reported to regulate and be regulated by IFN signaling (particularly via STAT phosphorylation) ${ }^{13,48}$, PD-L1 levels and STAT activation were examined after acquired PD-L1 treatment resistance and after IFN $\beta$ stimulation. First, PD-L1 was found to be significantly decreased in EMT6-PDR variants compared to $\mathrm{P}$ controls, and were found to remain decreased after i) IFN $\beta$-induced expression elevations, and ii) IFNAR1 knockdown (Fig 3f). Second, total STAT1 expression was also found to decrease expression in EMT6-PDR cells compared to P controls while total STAT3 levels remained unchanged (Fig 3g; blotting replicates in Appendix 1; densitometry to $\beta$-actin shown in Fig 3h). Despite this expression decrease, IFN $\beta$ treatment led to a significant increase in 
pSTAT1 and pSTAT3 (relative to total protein levels) in EMT6-PDR cells compared to P controls, an effect that was partially reversed in PDR-IFNAR1 KD cells (Fig 3i). As confirmation, pSTAT3 levels were also examined via ELISA assays which yielded similar findings (Fig 3j). Taken together, these results show that increased PTIS expression after acquired resistance can be further enhanced by IFN stimulation - particularly IFN $\beta$ - and regulated primarily via type I IFNcontrolled intracellular signaling in tumor cells.

\section{Persistent PD-L1 blockade in vitro sensitizes tumor cells to type I IFN stimulation of PTIS}

To examine whether IFN-regulated PTIS effects after acquired resistance could be directly linked to inhibition of PD-L1 on tumor cells, EMT6 cells were chronically treated with $\alpha$ PD-L1 (clone 80) or IgG in vitro for $>4$ weeks to generate EMT6-PVITRO and PDR VITRO cell variants (Fig 4a; schematic shown). Global transcriptomic changes found using RNAseq analysis revealed multiple genes to be up- or down-regulated (Fig 4b). While these gene changes were comparatively less robust than in in vivo-derived EMT6-P and PDR tumor tissue (Fig 4c) and found not to consistently differ between individual ISGs (data not shown), the PTIS signature was significantly enriched in EMT6-PDR VITRO cells compared to parental controls (Fig 4d). EMT6PDR VITRO cell variants were also found to be enriched for several IFN-associated gene sets from published datasets (Fig 4e) and in tumor tissues taken from durvalumab-treated NSCLC patients (Fig 4f; See Methods and Table S1). Critically, multiple ISGs of the PTIS (including IL6) were found to be significantly enhanced in EMT6-PDR VITRO cells following IFN $\beta$ stimulation compared to parental controls (Fig 4g: 4h shows heatmap summary), indicating that type I IFN-controlled secretory changes in the tumor can be, at least in part, a direct response to PD-L1 inhibition. For confirmation, we next tested whether similar results could be obtained using different cancer cell 
types and different PD-L1 inhibition strategies. First, using a $\alpha$ PD-L1 antibody (MIH5), we generated a mouse colorectal CT26-PDR VITRO cell line and found IL6 protein expression to significantly increase before and after IFN $\beta$-stimulation (Fig 4i). Second, using a PD-L1 knock-

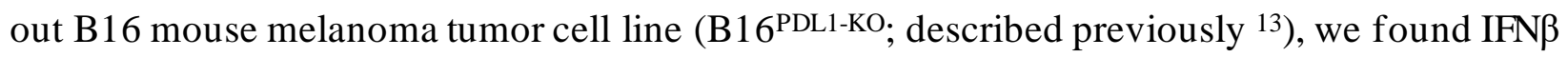
stimulation could also increase multiple ISGs (Fig 4j: 4k shows heatmap summary). Detailed analysis of IL6 in B16 was limited by low gene and protein expression levels (data not shown), though it is notable that similar trends were observed. Finally, to determine whether type I IFNenhanced PTIS changes are specific to the duration of treatment, we compared EMT6 cells exposed to $\alpha$ PD-L1 (clone 80 ) for long- (> 4 week) and short-term (1 week) periods. Results showed IFN $\beta$-stimulated IL6 expression to be significantly increased after longer, but not shorter, duration (Fig 4l). Taken together, these results show that persistent PD-L1 blockade can lead to tumor-specific adaptations involving intracellular PD-L1:IFN signaling that, in turn, can sensitize tumor cells to enhanced type I IFN-driven secretory changes.

\section{PDR-mediated immune-protection is IFN signaling-dependent}

Next, we assessed whether the cellular and secretory changes in PDR cells might influence (or be influenced by) immune cell populations typically part of the anti-tumor response. We first performed CIBERSORT/ImmuCC tissue deconvolution analysis to identify mouse gene scores representing immune cell populations in RNAseq data from EMT6-P and -PDR tumors (described in ${ }^{49,50}$ ). EMT6-PDR tumors had higher total immune scores (Fig 5a), with activated cytotoxic CD8+ T lymphocyte (CTL) and M2 macrophage scores significantly decreased (Fig 5b), indicating that anti-tumor immune response may be suppressed after resistance. Since a decrease in major histocompatibility complex class I (MHC-I) expression can help tumors evade cytotoxic 
CD8+ T lymphocytes (CTLs) and can be impacted by IFN-signaling 51 , MHC-I was examined by flow cytometry (Fig 5c;upper panel) and found to be significantly decreased in EMT6-PDR (Fig 5c;lower left panel), with this decrease reversed in EMT6-PDR-IFNAR1 ${ }^{\mathrm{KD}}$ cells (Fig 5c;lower right panel). Next, we tested whether secretory factors released from resistant cells may influence CTL proliferation and activation markers. To do this, conditioned media (CM) from EMT6-P/PDR cells was incubated with dissociated mouse splenocytes containing CD8+ T cells activated by $\alpha \mathrm{CD} 3$ and $\alpha \mathrm{CD} 28$ antibodies (Fig 5d; schematic shown). Significant decreases in CD8 T-cell proliferation were found to be induced by EMT6-PDR CM (Fig 5e), something that was reversed in CM from EMT6-PDR-IFNAR1 ${ }^{\mathrm{KD}}$ cells (Fig 5f). Next, CM from EMT6-PDR cells was found to decrease CD8+ T cells co-expressing markers for cytotoxicity such as Granzyme B and IFN $\gamma$ (both T-cell effector proteins capable of cell-killing ${ }^{52,53}$ ) and CD69, an early T-cell activation marker ${ }^{54}$, compared to unstimulated splenocytes and $\mathrm{P}$ controls (Fig 5g). This immunesuppressive effect was again found to be reversed in CM from EMT6-PDR-IFNAR1 ${ }^{\text {KD }}$ cells (Fig 5h). Next, we tested for tumor cell cytotoxicity in response following co-culture with activated CD8+ T-cells (Fig 5i; schematic shown) using flow cytometry (Fig 5j; representative images shown). Results show that tumor markers for apoptosis (annexin V) and cell death (7-AAD) were significantly decreased in EMT6-PDR cells compared to P controls (Fig 5k), with this effect reversed in EMT6-PDR-IFNAR1 ${ }^{\mathrm{KD}}$ cells and apoptosis increased (Fig 5l). In the same co-culture studies, CTLs were also measured by flow cytometry; however, consistent results were not observed which we attribute to complex direct tumor:splenocyte interactions that may influence the outcome (Fig S4a-e; See supplemental Results). Together, these results identify two significant 'immune protective' changes to the tumor that occur after acquired resistance to PDL1 inhibition. First, resistant cell secretory changes can suppress CD8+ T cell activation; and 
second, tumoral IFN-signaling is 'rewired' to limit antigen presentation and response to antiapoptotic stimuli.

\section{Inhibition of PTIS regulators selectively inhibits PDR tumor growth}

To examine whether immune-protective changes after acquired resistance to $\alpha$ PD-L1 inhibition can impact tumor growth in vivo, we first tested blocking individual PTIS molecules such as IL-6. In addition to IL-6 representing a PTIS protein consistently shown to be enhanced by IFNs in our PDR models, IL6 has also been shown to be a suppressor of anti-tumor immune responses and can improve PD-1 pathway inhibitor efficacy when targeted clinically and preclinically 4,55 . Following orthotopic implantation of EMT6-P and PDR cells, Balb/c mice treated with anti-mouse IL-6 ( $\alpha$ mIL-6) antibody showed a significant reductions in tumor growth only in the PDR variants, and not in the $\mathrm{P}$ tumor controls (Fig 6a-right, daily growth and area under the curve (AUC) analysis; 6a-left, treated vs untreated comparison). Next we tested whether blocking type I IFN-signaling control of the PTIS may have a more expanded impact on tumor growth after resistance, we implanted EMT6-P-and -PDR-IFNAR1 ${ }^{\mathrm{KD}}$ variants orthotopically into Balb/c mice (Fig 6b-right, daily growth and AUC analysis; 6b-left, treated vs untreated comparison). Notably, IFNAR1 knock-down in EMT6-P cells significantly increased tumor growth compared to $\mathrm{P}$ controls (Fig 6b-blue lines), providing confirmation of previous reports indicating that treatment-naïve tumor growth can be enhanced after type I IFN signaling blockade 56,57. Conversely, growth of EMT6-PDR-IFNAR1 KD variants were found to be significantly inhibited compared to EMT6-PDR tumors, demonstrating that type I IFN-enhanced PTIS confers a 'protective' effect that, when inhibited, has strong anti-tumor effects specific to $\alpha$ PD-L1 resistant cells (Fig 6b: red lines). AUC analysis of results in 6A-B confirmed the magnitude of IL6 and 
IFNAR1 inhibitory effect on EMT6-PDR tumors (Fig 6c). Interestingly, these PDR cell-specific effects may extend to inhibition of metastatic spread as post-mortem analysis at endpoint (Day 27 for $\alpha$ IL6, Day 26 for IFNAR1 ${ }^{\mathrm{KD}}$ ) showed enhanced inhibition of tumor skin/abdominal wall invasion after $\alpha$-mIL-6 (2.5-fold decrease in PDR vs 2-fold decrease in P tumors) and IFNAR1 ${ }^{\mathrm{KD}}$ (no invasion in PDR and 3.125-fold increase in P tumors) (Fig 6d). Taken together these results indicate that inhibition of PTIS and key regulators of its expression may provide enhanced benefit after acquired resistance to PD-L1 blockade. 


\section{Discussion}

A subset of cancer patients who are initially responsive to PD-L1 inhibitors will develop acquired resistance ${ }^{58,59}$. Mechanisms to explain why immunologically 'hot' tumors turn 'cold' remain complex as the microenvironment can adapt to treatment by relying on alternative checkpoints, inducing permanent $\mathrm{T}$ cell exhaustion, and recruiting/expanding an array of immunosuppressive cells - amongst many other changes attributed to host cell populations (reviewed in ref ${ }^{58,60}$ ). But there is increasing evidence that the tumor also adapts to PD-L1 blockade ${ }^{61,62}$. Here, we examined the consequences of prolonged PD-L1 inhibition in vivo and in vitro on tumor cells and identified a unique PTIS signature that was associated with acquired resistance, enriched for numerous ISGs, and tightly regulated by type I IFN signaling. These tumor intrinsic adaptations were found to protect tumor cells fromimmune mediated cytotoxicity directly, via decreased sensitivity to lymphocytic attack, and indirectly, via a suppression of $\mathrm{T}$ cell activation by the PTIS. Importantly, resistant tumors were found to be uniquely vulnerable to IFN signaling disruption which, when targeted in vivo, could reverse immune-protective effects and enhance tumor growth inhibition. Together, these findings suggest that a consequence of chronic PD-L1 blockade includes a tumor intrinsic secretory signature that may serve as a biomarker and molecular driver of acquired resistance in patients.

Studies examining the basic mechanisms of ICI-mediated tumor inhibition have mainly focused on the effects of blocking PD-L1 extrinsic functions that modulate immune cells such as T-, NK-, and myeloid cell populations, amongst several others ${ }^{63-65}$. Yet tumor intrinsic PD-L1 signaling is also impacted by therapeutic inhibition and can contribute to resistance. For instance, PD-L1 has been described as a 'molecular shield' as it can protect tumor cells from T cell cytotoxicity induced by various treatments ${ }^{17,66}$ and by IFNs $^{13,67}$. Indeed, Gato-Canas and 
colleagues found that type I IFN-induced cytotoxicity can be controlled by conserved motifs of PD-L1 cytoplasmic domains (RMLDVEKC and DTSSK), which block STAT3/Caspase 7 cleavage $^{13}$. Our results show prolonged PD-L1 blockade can add to this immune-protective effect by altering IFN:PDL1 intracellular crosstalk which, in turn, can drive an IFN-enriched secretory program that contributes to treatment resistance. Indeed, we found PDR cells were less susceptible to activated splenocyte mediated cytotoxicity, had increased activation of STAT3/1-controlled anti-apoptotic programs after IFN stimulation ${ }^{68}$, and had a secretory profile enriched for immunosuppressing factors such as SERPINB9, which serve as an endogenous inhibitor of granzyme $\mathrm{B}^{26,69}$. This latter finding may explain why conditioned media from PDR cells was able suppress several markers of $\mathrm{T}$ cell activation and raises the question of whether inhibition of one protective mechanism (PD-L1) may be compensated for by other protective mechanisms (i.e., other ISGs). Such considerations may depend on several variables known to affect efficacy, including antibody ty $\mathrm{pe}^{70}$, treatment $\mathrm{dose}^{13}$, and treatment duration. On this latter topic, our results show that the IFN-enhanced PTIS could be replicated, at least in part, by direct antibody inhibition or PD-L1 knockout in vitro, but only after sustained inhibition periods longer than 1 month. This may be relevant to patients who develop acquired resistance to PD-L1 inhibition and receive treatment for up to two years, depending on trial protocols and tumor response ${ }^{44,71}$. Because patient sub-sets have been shown to have highly durable responses to PD-1 pathway inhibition even after treatment discontinuation 72,73 , our results may support rationale to avoid "over-treatment" with $\mathrm{ICI}^{74,75}$.

An important implication for these studies involves the potential use of PTIS as a biomarker of acquired PD-L1 treatment resistance. Transcriptomic analysis comparing tumor tissues has shown ISGs can increase in both tumor and non-tumor cell populations before and after 
ICI treatment in patients ${ }^{27,41,43,76}$. Here we examined 5 preclinical and 3 clinical datasets and found the PTIS to be enriched in tumors initially sensitive to $\alpha$ PD-L1 treatment. This would suggest that assessing the PTIS signature in patient biopsies after treatment may have utility as a predictor for sensitivity levels, particularly given our results showing significant downregulation of PTIS in models of innate resistance. This may be feasible to test in tissues obtained after neoadjuvant PDL1 inhibition such as in a current trial involving cutaneous melanoma patients treated with atezolizumab for 6 weeks (NCT04020809). It is also possible that monitoring individual PTIS factors might have predictive value in patients after treatment, though this is likely to be complex owing to the many functions of several proteins. For instance, secreted circulating factors such as IL6, CXCL9, and CXCL10 have been found to increase in patients after PD-1 pathway inhibition and correlate with tumor stabilization/shrinkage, as measured by objective response rates (ORR) 6,77-79. But few studies have yet to assess cytokine changes in patients after prolonged treatment durations that assess progression-free and overall survival (PFS/OS) outcomes ${ }^{7,80}$. Results from such studies may be mixed as IL-6 at baseline can correlate with improved initial response to nivolumab in a phase II trial for advanced melanoma (measured by ORR) ${ }^{77}$, but also predict for worse long-term outcome to PD-1/L1 inhibitors in NSCLC (measured by PFS) ${ }^{81}$. Furthermore, expression of some PTIS factors have been linked to both tumor-promoting and tumor-inhibiting immune responses depending on context. An example includes CXCL9 and CXCL10, which we found to be increased in IFN $\beta$-stimulated PDR cells; and thus far, been primarily associated with anti-tumor immune responses, including trafficking of cytotoxic $\mathrm{T}$ cells ${ }^{82-84}$. But in different disease types and tumor models, these cytokines can also be associated with recruitment of immune suppressive/tumor promoting T-regulatory cells ${ }^{85-87}$. In this regard, it is of particular interest that the PTIS was found to be enriched in non-tumor populations such as macrophages and 
T cells of merkel cell carcinoma patients treated with avelumab (Fig 2E) - suggesting PD-L1 inhibition can likely induce 'off-target' host effects ${ }^{19}$. This may portend to the PTIS playing a role in immune related adverse events (irAEs) known to be induced by various ICI treatments and associated with the systemic increase in cytokines, such as IL6, IL1-RA, and CXCL10 ${ }^{88}$. Indeed, PD-1 targeting agents have been known to induce cytokine release syndrome, which is an adverse effect characterized by fever, myalgias, malaise, and high levels of cytokines including IL6 and IFNs $^{89,90}$. Assessment of the PTIS as a potential cause or consequence of these processes requires further investigation.

The impact of IFN signaling on tumor growth and overall efficacy of ICIs can have opposing, and of ten contradictory, effects depending on several factors that include the type of cell, the duration of IFN exposure, and the stage of tumor progression. Indeed, IFNs have been shown to have both stimulatory and inhibitory effects on tumor growth that can influence response to ICI treatment. For inhibitory effects, IFNs (mostly IFN $\gamma$ ) are integral to anti-tumor immunity by driving antigen presentation ${ }^{44}$ and chemokine secretion ${ }^{91}$ that are typically part of the immuneediting process in normal physiological conditions. IFNs may improve ICI responses as loss of function mutations in IFN signaling components (JAK1/2) have been noted in melanoma patients who have relapsed after pembrolizumab treatment ${ }^{92}$, and knockout of IFN pathway mediators such as Jak1, Stat1, Ifngr1 in tumors can weaken ICI treatment efficacy 93,94 . For IFN tumor stimulatory effects, Benci and colleagues demonstrated that ICIs can induce an enhanced expression of tumor cell ISGs transcribing additional T-cell co-inhibitory ligands (e.g. TNFRSF14, LGALS9) where blockade of type I and II IFN signaling could reverse this effect and improve ICI responses ${ }^{20}$. Additional tumor ISGs regulated by type I IFNs such as NOS2 25 and CD3827 can also have immune-suppressive effects and mediate acquired resistance to PD-1 pathway blockade. 
Furthermore, high levels of type II IFN induced by combined PD-1/CTLA-4 blockade can also mediate deletion of anti-tumor T cells under low-tumor burden settings ${ }^{95}$.

Due to these opposing roles for IFN, one question is whether negative consequences of IFN can be specifically targeted, without negating the positive IFN signaling effects. In this regard, Benci and colleagues proposed that ISGs promoting ICI resistance may be more associated with tumor cell expression ${ }^{21}$ and after chronic exposure to IFNs ${ }^{20}$, compared to the largely positive effects of IFNs on immune cells and after acute IFN exposure. In these studies, sequential treatment of an ICI followed by a JAK inhibitor was found to sensitize IFN-driven resistant tumors to ICI treatment ${ }^{20}$. Such treatment strategies are now being tested in clinical trials (NCT03425006). A second ISG targeting approach aims to block the individual ISG itself which, theoretically, could avoid suppressing IFNs anti-tumor functions. In our study, both approaches were evaluated. In the first, IFNAR1 knockdown in PDR cells was found to effectively reverse IFN $\beta$-mediated PTIS factor increases and reverse direct (and indirect) immune suppressive effects in co-culture studies with mouse splenocytes. This may explain why PDR-IFNAR1 KD tumors were found to grow slower than PDR tumors in vivo, and highlight the unique vulnerability induced by constant PDL1 blockade. However, our results also show that IFNAR1 KD tumors grew much faster than parental controls, emphasizing the sometimes contradictory role of IFNAR signaling in cancer controlling immunosurvellance ${ }^{28}$ may be exacerbated by PD-L1 treatment and, as a consequence, introduce potential challenges of intracellular IFN signaling inhibition strategies. In our second approach, we targeted IL-6, which is a specific component of the PTIS that was consistently increased in PDR cells and then further enhanced after IFN $\beta$ stimulation. IL6 is known to activate a multitude of tumor promoting effects including (i) enhancing ex pression of pro-angiogenic factors in tumors cells (e.g. VEGF, IL1 $\beta$, IL896), (ii) suppressing antigen presentation from 
dendritic cells ${ }^{97}$, (iii) promoting pro-tumorigenic macrophage phenotypes ${ }^{98}$, (iv) suppressing antitumor functions of CD4+ T cells ${ }^{99}$, amongst others ${ }^{100}$. Trials are currently underway testing IL6 and PD-1 pathway inhibitor combinations for improved anti-tumor efficacy and irAEs (NCT03999749, NCT04258150)101,102. Our results show that IL6 inhibition can lead to enhanced growth suppression in PDR tumors supporting a rationale for IL6:PD-L1 combination/sequencing strategies. However, it should be noted that these effects were largely modest, suggesting targeting multiple PTIS factors simultaneously may yield more robust outcomes. In favor of this, preclinical models involving inhibition of CCL2 ${ }^{103}$, CCL5 ${ }^{104}$, NOS2 ${ }^{25}$, and SERPINB9 ${ }^{26}$ pathways have all shown benefits when combined with various ICIs and may be explored to improve IL6 inhibitory strategies after PD-L1 treatment failure.

Taken together, our results show that tumor cells following acquired resistance to PD-L1 blockade can express an ISG enriched secretory profile associated with diminished sensitivity to immune cell cytotoxicity. Therapeutic approaches involving inhibition of PTIS components or IFN regulators may have enhanced benefit after resistance to PD-L1 inhibition. 


\section{Acknowledgements}

We would like to thank A. Haninec for helpfulcomments; and A. Tracz, S. Grant, A. June for their technical support. Select cell lines used in this study were kind gifts from various laboratories. These include EMT6 (A. Gukov) and B16 (D. Escors). We thank M. Azuma for providing MIH5 hybridoma for antibody production (see Methods) and M. Oberst at AstraZeneca for providing the Clone 80 antibody.

Funding: This work used shared resources supported by the Roswell Park Comprehensive Cancer

Center (RPCCC) Support Grant from the National Cancer Institute (NCI) (P30CA016056). This work was supported by grants to JMLE from the American Cancer Society (ACS) via a Research Scholar Grant (RSG-18-064-01-TBG) and Roswell Park Alliance Foundation (RPAF); and to YS from NCI F30 CA243281. Opinions, interpretations, conclusions and recommendations are those of the author and are not necessarily endorsed by the RPAF, NCI, or ACS.

\section{Author Contributions:}

Conceptualization, YS, JMLE; Methodology, YS, MD, MM, KE, SB, JMLE; Investigation, YS, MD, MM, WH, AD, JMLE; Formal Analysis, YS, MM, MD, JMLE; Visualization, YS, MM, JMLE; Supervision, JMLE; Funding Acquisition, JMLE; Writing - Original Draft, YS, JMLE; Writing - Review and Editing, YS, MD, MM, JMLE.

\section{Declaration of Interests:}

None 


\section{Methods}

\section{CONTACT FOR REAGENT AND RESOURCE SHARING}

Further information and requests for resources and reagents should be directed to and will be fulfilled by Lead Contact, John M.L. Ebos (John.Ebos@RoswellPark.org).

\section{Cell lines}

Cell used in this study include: Mouse mammary carcinoma EMT6 (from A. Gudkov, Roswell Park Comprehensive Cancer Center, RPCCC), colorectal carcinoma CT26 (A. Gudkov), mouse kidney RENCA (from R. Pili, RPCCC) and melanoma B16 control and PD-L1 knockout cells (from David Escors, Navarrabiomed as described previously ${ }^{13}$ ). Cells were maintained in RPMI (Corning cellgro \#10-040-CV) supplemented with 5\% v/v FBS (Corning cellgro; 35-010-CV). All cells were maintained at $37^{\circ} \mathrm{C}$ with $5 \% \mathrm{CO} 2$ in a humidified incubator.

\section{Drug and recombinant protein concentrations}

IgG1 (NIP228, AstraZeneca), IgG2a (I-1177, Leinco Technologies Inc), aPD-L1 (clone 80, AstraZeneca), $\alpha$ PD-L1 (MIH5, from M. Azuma, Tokyo Medical and Dental University ${ }^{105}$ ) and anti-IL6 (BE0046/MP5-20F3, BioXCell) were prepared as follows: For in vivo experiments: $\alpha \mathrm{PD}-$ L1 (Clone80) and anti-IL6 (MP5-20F3) were diluted in PBS and administered by intraperitoneal injection at $(250 \mu \mathrm{g} / \mathrm{mouse} / 3$ days $)$ or $(100 \mu \mathrm{g} / \mathrm{mouse} / 3$ days $)$ respectively. Tumor-related differences between any vehicle or IgG groups were not observed. In vitro, IgG(NIP228 or I-1 177) and $\alpha$ PD-L1 (clone80 or MIH5) in PBS was directly added to media for maintenance at a concentration of $0.5 \mu \mathrm{g} / \mathrm{ml}$; anti-IL6 was used at a concentration of $10 \mu \mathrm{g} / \mathrm{ml}$; recombinant IFN- 
alpha-2 (50525-MNAY, Sino Biological), IFN-beta (50708-MCCH, Sino Biological), IFN $\gamma$ (31505, Peprotech) were used at 10ng/ml.

\section{shRNA knockdown studies}

For production of IFNAR1 knockdown lentivirus, pLKO.1-puro shRNA plasmid DNA was isolated from bacteria glycerol stocks (TRCN0000301483; Sigma Aldrich) using E.Z.N.A.® Plasmid Mini Kit I (Omega Bio-tek, Inc.). To produce lentiviral media, 293T cells were transiently co-transfected with DNA from the lentiviral pLKO.1-puro shRNA plasmid and psPAX2 and pMD2.G packaging plasmids using LipoD293 ${ }^{\mathrm{TM}}$ Transfection Reagent (SignaGen Laboratories.) Conditioned media containing virions was harvested after 24 and 48 hours, filtered through a 0.45 $\mu \mathrm{m}$ membrane, and used to infect EMT6-P and PDR cells. Cells were infected with the shRNA and vector controls by spin inoculation at $600 \times \mathrm{g}$ for $45 \mathrm{~min}$ at room temperature in the presence of $5 \mathrm{ug} / \mathrm{ml}$ polybrene. Viruses were removed after an additional $6 \mathrm{hr}$ incubation at $37^{\circ} \mathrm{c} / 5 \% \mathrm{CO} 2$ and cell culture media was replaced. Puromycin selection was then conducted for 2 weeks at $2 \mu \mathrm{g} / \mathrm{ml}$ until stably infected cells were generated. Knockdown was confirmed via flow cytometry analysis.

\section{Mouse tumor models}

\section{$\underline{\text { Study Approval }}$}

Animal tumor model studies were performed in strict accordance with the recommendations in the Guide for Care and Use of Laboratory Animals of the National Institutes of Health and according to guidelines of the IACUC at RPCCC (Protocol: 1227M).

Orthotopic Tumor Implantations 


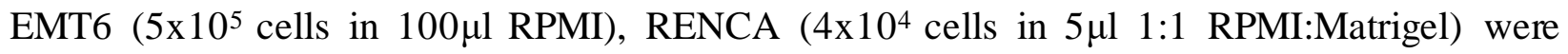
implanted orthotopically into the right inguinal mammary fad pad or left kidney subcapsular space respectively in 6-8 week old female Balb/c mice. Isoflurance (anesthesia) and buprenorphrine (analgesic) were used during all surgical implantations. Mammary fat pad tumors were measured using Vernier calipers and volumes were calculated using the formula (width ${ }^{2} \mathrm{x}$ length) $\mathrm{x} 0.5$. Kidney luciferase expressing tumors were assessed for bioluminescence activity bi-weekly. All animals were assessed 2-3 times daily by veterinary staff or personnel approved by IACUC for pre-defined endpoints. Institutional endpoints included primary tumor-based morbidities (>2000 $\mathrm{mm}^{3}$ volume) and metastasis related morbidities (labored breathing, $20 \%$ weight loss, cachexia, limb paralysis). All mice were randomized before implantation.

\section{Resistance cell derivation and maintenance}

For in vivo-derived PDR cell variants, mice were orthotopically implanted with EMT6 or RENCA and treated with $\alpha$ PD-L1 (Clone80) until institutional endpoint. For both EMT6 and RENCA, parental $(\mathrm{P})$ cell lines were obtained from IgG-treated mice and used as controls. All variants were selected from primary tumors which were minced, enzymatically digested (Miltenyi Biotics; 130095-929), and then placed in RPMI media (supplemented with 5\% v/v FBS, 100IU/ml penicillin and $1000 \mu \mathrm{g} / \mathrm{ml}$ streptomycin) with $\mathrm{IgG}$ (NIP288) for P variants or with $\alpha \mathrm{PD}-\mathrm{L} 1$ (clone80) $(0.5 \mu \mathrm{g} / \mathrm{ml})$ for PDR variants. Antibiotics were then removed 1 week after in vivo cell selection. For derivation of PDR ${ }^{\text {VITRO }}$ cell variants, EMT6 and CT26 cells were treated with $\alpha$ PD-L1 antibodies (clone 80 at $0.5 \mu \mathrm{g} / \mathrm{ml}$ or MIH5 at $0.5 \mu \mathrm{g} / \mathrm{ml}$, respectively) for $>4$ weeks.

\section{Cell proliferation assay}


Proliferation was examined using CellTiter 96 Aqueous Non-Radioactive cell proliferation (MTS) assay (Promega; G1112). For 5 day growth studies, 200 cells/well were plated in 48 -well plates. The next day, cells were treated with recombinant IFNs or anti-IL6. Treatments were replaced every 2 days or removed daily for MTS measure of viability. RPMI $+5 \%$ FBS was mixed with MTS per manufacturer instructions and added to cells at timepoints. After 2 hour incubation, optical density was measured at a wavelength of 490nm (Bio-Rad xMark).

\section{RNA isolation}

Cells were plated at 80,000 cells/well in a 6 well plate with corresponding treatments as indicated. 48 hours later, total RNA was isolated using QIAshredder (QIAGEN; 79654) and RNase mini kit (QIAGEN; 74104). Genomic DNA was then digested using DNaseI (QIAGEN; 79254) per manufactuer instructions. RNA concentration was determined using nanodrop 2000c (Thermo Scientific) before RNAseq and PCR analysis.

\section{qRT-PCR}

For reverse transcription using iScriptcDNA synthesis kit(Bio-rad; 170-8891), $1 \mu \mathrm{g}$ RNA was used according to the manufacturer's instructions. qRT-PCR was performed using iTaq SYBR Green Supermix (Bio-rad;1725121). Thermocycling parameters were: $10 \mathrm{~min}$ at $95 \mathrm{oC}, 15 \mathrm{sec}$ at $95 \mathrm{oC}$, 40 cycles at $95 \mathrm{oC}$ for $15 \mathrm{sec}$ at $95 \mathrm{oC}$ and $1 \mathrm{~min}$ at $60 \mathrm{oC}, 1 \mathrm{~min}$ at $95 \mathrm{oC}$, followed by a melting curve: 55 to $95 \mathrm{oC}$ with increments of $0.5 \mathrm{oC}$ for $5 \mathrm{sec}$. Relative gene expression was calculated using the formula 2-[CT(House Keeping Gene)-Ct(Gene of Interest)], with CT representing the fixed threshold cycle value for fluorescent signal. Gapdh and Actb were used for housekeeping genes. Oligonucleotides were purchased from Integrated DNA Technologies (IDT). 


\section{Proteome profiler array}

Cells were lysed with lysis buffer 17 (R\&D Systems; 895943) supplemented with protease cocktail (Fisher Scientific, PI78430). Total protein levels were quantified with DC protein assay (Bio-Rad; 500-0112). 200 $\mu \mathrm{g}$ of total mouse protein samples were analyzed respectively with a Mouse XL Cytokine Array Kit (R\&D Systems; ARY028) per manufacturer instructions. Membranes were exposed to X-ray films, which were imaged (digitized) with ChemiDoc System (Bio-Rad) and analyzed with Image Lab Software (Bio-Rad).

\section{ELISA analysis}

Cells were lysed with lysis buffer I (20mM Tris (pH7.5), 127mM NaCl, $10 \%$ Glycerol, $1 \%$ v/v NP40 (Igepal), 100mM NaF, 1 mM Na3VO4) and protein concentrations were quantified with DC protein assay. For conditioned media collection, cells were counted for normalization. IL-6, phospho-STAT3, and PD-L1 were measured using mouse IL6 ELISA (431304, Biolegend), mouse phospho-STAT3 ELISA (7300C), and mouse PD-L1 Duoset ELISA (DY1019-05).

\section{Western blot analysis}

Cells were lysed with lysis buffer II (50mM Tris (pH8), 2\% w/v SDS, 5mM EDTA, 3mM EGTA, $25 \mathrm{mM} \mathrm{NaF}, 1 \mathrm{mM} \mathrm{Na}_{3} \mathrm{VO}_{4}$ ) supplemented with Halt ${ }^{\mathrm{TM}}$ protease inhibitor (Thermo Fischer Scientific 78429). Lysates were sonicated for 2 seconds and total protein concentration was quantified with DC protein assay. Proteins samples were prepared with 1/5 volume of 5x SDSPAGE sample buffer (250mM Tris pH6.8, $10 \% \mathrm{w} / \mathrm{v}$ SDS, $25 \%$ v/v glycerol, 500mM DTT, and bromophenol blue). Proteins (40 $\mu \mathrm{g}$ per lane) were resolved by SDS-PAGE, electrotransferred to 
Immobilon-P membrane, and incubated with a primary antibody diluted as recommended by the manufacturer. Membranes were then probed with a horseradish peroxidase-conjugated secondary antibody (Promega W4011 and W4021) and protein signals were developed using the Pierce ECL Western blotting substrate (Thermo Scientific; 32106). X-ray films were imaged (digitized) with ChemiDoc System and analyzed with Image Lab Software. Primary antibodies were purchased from Cell signaling (phospho-STAT1 Tyr701 9167S, STAT1 14994, phospho-STAT3 Tyr705 9145T, STAT3 9139T) and Sigma Aldrich ( $\beta$-actin, A5441).

\section{Splenocyte Division and Activation Assays}

Spleens were harvested from Balb/c mice, mechanically dissociated by passing through a $70 \mu \mathrm{m}$ filter, and collected in complete RPMI media (supplemented with 10\% heat-inactivated FBS, $1 \%$ non-essential amino acids, $1 \%$ sodium pyruvate, $1 \%$ penicillin/streptomycin, and $0.1 \% \beta$ mercaptoethanol). Splenocytes were then treated with RBC lysis buffer (Biolegend, 420301) and incubated overnight. The next day, splenocytes were stained with CFSE (Biolegend, 423801), stimulated with anti-mouse CD3 (Biolegend, 100202) and CD28 (Biolegend, 102102) according to manufacturer recommendations. To generate conditioned media, 7 x $10^{6}$ tumor cells were plated with $10 \mathrm{ml}$ of RPMI (supplemented with 5\% FBS) in a 10-cm dish. After 72 hours, conditioned media was collected and passed through a $0.2 \mu \mathrm{m}$ filter. Splenocytes were then plated in a 1:1 ratio of complete RPMI:conditioned media at 400,000 cells per well in a 96 -well plate. After 72 hours of incubation splenocytes were treated for 5 hours with activation cocktail with brefeldin A1 (Biolegend, 423303) before staining for CD45 (Biolegend, 103128), CD8b.2 (Biolegend, 140416), Granzyme B (Biolegend, 515408), IFN $\gamma$ (Biolegend, 505808), and CD69 (Biolegend, 104514) according to manufacture instructions. 
For tumor cell co-culture experiments, $1 \times 10^{4}$ tumor cells were plated in a 24 -well plate per well and allowed to adhere overnight. The next day, $1 \times 10^{6}$ stained and stimulated splenocytes were added per well. After 72 hours of incubation, splenocytes were processed as described above and adherent tumor cells were concurrently collected for Annexin V (Biolegend, 640920) and 7-AAD staining (Biolegend, 420404) via flow cy tometry according to manufacturer instructions.

\section{Flow cy tometry analysis of cell surface proteins}

Cells were plated at 80,000 cells/well in a 6 well plate. After two days, cells were collected by accutase (Biolegend, 423201) and analyzed by flow cytometry for $\mathrm{H}-2 \mathrm{~Kb} / \mathrm{H}-2 \mathrm{Db}$ (Biolegend, 114613) expression according to manufacture instructions.

\section{Whole transcriptome expression analysis}

RNA sequencing for tumor tissue-derived EMT6-PDR, and tumor cell line-derived EMT6PDR $^{\text {VITRO }}$ and RENCA-PDR cells, were performed utilizing the Genomic shared resource at RPCCC as previously described ${ }^{106}$. Sequencing library were prepared with TruSeq Stranded mRNA kit (Illumina Inc), from $1 \mu \mathrm{g}$ total RNA, according to manufacturer's instructions. PolyA selection, RNA purification, fragmentation and priming for cDNA synthesis was performed. Using random primers, fragmented RNA was then reverse transcribed into first-strand cDNA. RNA template was then removed, a replacement strand was synthesized and dUTP was incorporated in place of dTTP to generate ds cDNA. ds cDNA was separated from second-strand reaction mix using AMPure XP beads (Beckman Coulter) resulting in blunt-ended cDNA. One 'A' nucleotide was added to the 3 ' ends of the blunt fragments. Multiple indexing adapters, containing one ' $\mathrm{T}$ ' nucleotide on the 3' end of the adapter, were ligated to the ends of the ds cDNA, preparing them 
for hybridization onto a flow cell. Adapter ligated libraries were amplified by PCR, purified using Ampure XP beads, and validated for appropriate size on a 4200 TapeStation D1000 Screentape (Agilent Technologies, Inc.). The DNA libraries were quantitated using KAPA Biosystems qPCR kit, and were pooled together in an equimolar fashion, following experimental design criteria. DNA library pool was denatured and diluted to $2.4 \mathrm{pM}$ with $1 \%$ PhiX control library added. The resulting pool was then loaded into the appropriate NextSeq Reagent cartridge, as determined by the number of sequencing cycles desired, and sequenced on a NextSeq500 following the manufacturer's recommended protocol (Illumina Inc.). Sequencing quality control was assessed using FASTQC v0.11.5 (http://www.bioinformatics.babraham.ac.uk/projects/fastqc/). Reads were aligned to the mouse genome GRCM38 M16 (genocode) using STAR v2.6.0a ${ }^{107}$ and postalignment quality control was assessed using RSeQC v2.6.5 ${ }^{108}$. Aligned reads were quantified using RSEM v1.3.1 ${ }^{109}$. Counts from RSEM were then filtered and then upper quartile normalized using R package edgeR. Data from RENCA and EMT6 studies were deposited in GEO (accession number: Pending).

\section{Gene Ontology Analysis/Cytoscape}

Differentially expressed genes with products located in extracellular regions were identified using gene ontology databases (GO:00005576) as previously described ${ }^{19,110}$. GO Biological processes terms were then assessed using ClueGo via Cytoscape v3.7.2 and significantly enriched terms and corresponding Kappa scores were plotted based on p-values.

\section{Gene Set Enrichment Analysis}


Gene set enrichment analysis (GSEA) was conducted to assess comparisons for molecular pathways and gene set correlations. A rank list was first generated using log2(fold change) gene expression data obtained from limma anlaysis. GSEA-Preranked was then conducted using a geneset permutation type with 1000 random permutations to obtain normalized enrichment scores (NES) and false discovery rate (FDR) q-values.

\section{Interferome Analysis}

Genes of interest were assessed in the Interferome Database (http://www.interferome.org/interferome/home.jspx) ${ }^{35}$ to examine for evidence of regulation by IFN signaling. Parameters interferome type, subtype, treatment concentration, treatment time, in vivo/in vitro, species, system, organ, cell, cell line, normal/abnormal were set to "any", fold-change thresholds were set to 1.5 .

\section{Identification of PTIS}

A preliminary $\alpha$ PD-L1 Treatment Induced Signature (PTIS) was identified by compiling upregulated and downregulated targets obtained from transcriptomic (RNAseq of PDR tumor tissues). PTIS was then compiled from targets confirmed in two or more transcriptome or proteomic assays of EMT6-PDR tumors (RNAseq, qRT-PCR, Cytokine array, and ELISA).

\section{CIBERSORT/ImmuCC Analysis}

Cibersort tissue deconvolution was performed using the ImmuCC signature to obtain absolute score for various cell types ${ }^{49,50,111}$. From 25 immune cell type signatures, no values were detected 
for eosinophil cells, CD4 Memory T cells, Neutrophil cells, and Plasma Cells; and thus, were excluded for quantification and analy sis.

\section{Confirmation of PTIS signature enrichment in published datasets}

Previously published clinical and preclinical datasets derived from studies after treatment or after acquired resistance to PD-L1 inhibition were obtained from the Gene Expression Omnibus (GEO, www.ncbi.nlm.nih.gov/geo/) or database of Genotypes and Phenotypes (dbGaP, https://www.ncbi.nlm.nih.gov/gap/).

Sceneay et al. 2019 (GSE130472): In this study, whole tissue RNA-seq (Illumina NextSeq500 with paired-end 75bp reads) were performed on 4T1 orthotopically implanted mammary tumors in 8-12 week (young; responsive) or $>12$ months (old, nonresponsive) Balb/c mice treated with $\alpha$ PD-L1 (clone 10F.9G2) or isotype (CloneLTF-2). Tumors were collected when caliper volumes were no larger than $\sim 150 \mathrm{~mm}^{3}$ after 3 doses of antibody treatment.

Lan et al. 2018 (GSE107801): In this study,_whole tissue RNAseq was (Illumina Hiseq 2500) conducted on EMT6 orthotopically implanted mammary tumors in Balb/c mice treated with $\alpha \mathrm{PD}-\mathrm{L} 1$ or isotype control. Tumors were collected 6 days after 3 doses of treatment given daily starting 20 days after implantation.

Efremova et al. 2018 (GSE93017): In this study, whole tissue RNAseq(Ion Torrent Proton) was conducted on MC38 subcutaneously implanted tumors in C57B1/6 mice treated with $\alpha$ PD-L1 (Clone10F.9G2) or isotype (Clone LTF-2) every 3-4 days until day 14 after implantation.

Gettinger et al. 2018 (phs001464.v1.p1): In this study, pre-treatment and posttreatment/acquired resistant biopsies were obtained from patients receiving various immune checkpoint inhibitor treatments (PD-L1, PD-1, CTLA-4 targeted therapies) for RNA-seq analysis 
(Illumina HiSeq2500) from formalin fix paraffin embedded samples. For validation analysis, all pretreatment samples were compared to samples after acquired resistance to PD-L1 inhibition. Note: the name of the PD-L1 inhibitor was not provided in this publication.

Paulson et al. 2018 (GSE117988, GSE118056): In this study, single cell RNAseq analysis on tumor tissues were conducted on an untreated patient biopsy (Illumina HiSeq 2500) and a patient biopsy at acquired resistance (unmatched) after $\alpha$ PD-L1 (avelumab), MCPyV-specific T cells, and radiation (Illumina NovaSeq 6000). Processed data were obtained from the GEO database. R packages SingleCellExperiment ${ }^{112}$, scater ${ }^{113}$, limma ${ }^{114}$, and Rtsne were used for analysis. Counts were quartile normalized and converted to counts per million (CPM). Clustered enrichment analysis was then conducted using markers PTRC/CD45 (tumor cells), CD3D (T cells), and CD68 (Macrophages) similar to previously described ${ }^{45}$.

PTIS signature expression levels in these datasets were compared by average counts per million (CPM) levels or GSEA enrichment (defined by FDR $\leq 0.25$ ).

\section{Statistical analysis}

Analysis was conducted using the GraphPad Prism software package v 8.4.0 (GraphPad sof tware Inc., San Diego, CA) and R v3.6.0 through RStudio v1.1.463(Integrated Development for R; RStudio, Inc., Boston, MA URL http://www.rstudio.com/). For in vivo studies, results are represented as mean \pm standard deviation $(\mathrm{SD})$ or standard error of mean (SEM), as indicated. Kaplan-Meier methods were utilized for analysis of percent to institutional endpoint curves. Fold change differences between treatment control groups were assessed via two-way ANOVA. For all results, comparisons between two groups were made with Student's two-tailed unpaired t-test, whereas one-way ANOVA was used for comparison of more than two groups. Tumor volume and 
bioRxiv preprint doi: https://doi.org/10.1101/2021.07.01.450417; this version posted July 1, 2021. The copyright holder for this preprint (which was not certified by peer review) is the author/funder. All rights reserved. No reuse allowed without permission.

bioluminescence measurements were compared for specified time points. A minimum FDR value of 0.25 was use for GSEA analysis (as indicated as described by the user guide) and significance level of 0.05 was used for all other analyses. 


\section{References}

1 Kerbel, R. S. \& Ebos, J. M. Peering into the aftermath: The inhospitable host? Nat.Med. 16, 1084-1085 (2010).

2 Ebos, J. M. Prodding the Beast: Assessing the Impact of Treatment-Induced Metastasis. Cancer Res 75, 3427-3435, doi:10.1158/0008-5472.CAN-15-0308 (2015).

3 Madden, E. C., Gorman, A. M., Logue, S. E. \& Samali, A. Tumour Cell Secretome in Chemoresistance and Tumour Recurrence. Trends in cancer 6, 489-505, doi:10.1016/j.trecan.2020.02.020 (2020).

4 Tsukamoto, H. et al. Combined Blockade of IL6 and PD-1/PD-L1 Signaling Abrogates Mutual Regulation of Their Immunosuppressive Effects in the Tumor Microenvironment. Cancer research 78, 5011-5022, doi:10.1158/0008-5472.CAN-18-0118 (2018).

5 Sanmamed, M. F. et al. Changes in serum interleukin-8 (IL-8) levels reflect and predict response to anti-PD-1 treatment in melanoma and non-small-cell lung cancer patients. Ann Oncol 28, 1988-1995, doi:10.1093/annonc/mdx190 (2017).

6 Naqash, A. R., Yang, L. V., Sanderlin, E. J., Atwell, D. C. \& Walker, P. R. Interleukin-6 as one of the potential mediators of immune-related adverse events in non-small cell lung cancer patients treated with immune checkpoint blockade: evidence from a case report. Acta Oncol 57, 705-708, doi:10.1080/0284186X.2017.1406668 (2018).

7 Bridge, J. A., Lee, J. C., Daud, A., Wells, J. W. \& Bluestone, J. A. Cytokines, Chemokines, and Other Biomarkers of Response for Checkpoint Inhibitor Therapy in Skin Cancer. Front Med (Lausanne) 5, 351, doi:10.3389/fmed.2018.00351 (2018).

8 Wei, S. C., Duffy, C. R. \& Allison, J. P. Fundamental Mechanisms of Immune Checkpoint Blockade Therapy. Cancer Discov 8, 1069-1086, doi:10.1158/2159-8290.CD-18-0367 (2018).

9 Ribas, A. \& Wolchok, J. D. Cancer immunotherapy using checkpoint blockade. Science 359, 1350-1355, doi:10.1126/science.aar4060 (2018).

10 Chang, C. H. et al. Metabolic Competition in the Tumor Microenvironment Is a Driver of Cancer Progression. Cell 162, 1229-1241, doi:10.1016/j.cell.2015.08.016 (2015).

11 Clark, C. A. et al. Tumor-Intrinsic PD-L1 Signals Regulate Cell Growth, Pathogenesis, and Autophagy in Ovarian Cancer and Melanoma. Cancer Res 76, 6964-6974, doi:10.1158/0008-5472.CAN-16-0258 (2016).

12 Wu, X. et al. Targeting B7-H1 (PD-L1) sensitizes cancer cells to chemotherapy. Heliyon 4, e01039, doi:10.1016/j.heliyon.2018.e01039 (2018).

13 Gato-Canas, M. et al. PDL1 Signals through Conserved Sequence Motifs to Overcome Interferon-Mediated Cytotoxicity. Cell Rep 20, 1818-1829, doi:10.1016/j.celrep.2017.07.075 (2017).

14 Wang, S. et al. Programmed death ligand 1 promotes lymph node metastasis and glucose metabolism in cervical cancer by activating integrin beta4/SNAI1/SIRT3 signaling pathway. Oncogene 37, 4164-4180, doi:10.1038/s41388-018-0252-x (2018).

$15 \mathrm{Du}, \mathrm{W}$. et al. KPNB1-mediated nuclear translocation of PD-L1 promotes non-small cell lung cancer cell proliferation via the Gas6/MerTK signaling pathway. Cell Death Differ 28, 1284-1300, doi:10.1038/s41418-020-00651-5 (2021). 
16 Feng, D. et al. BRAF(V600E)-induced, tumor intrinsic PD-L1 can regulate chemotherapyinduced apoptosis in human colon cancer cells and in tumor xenografts. Oncogene 38, 6752-6766, doi:10.1038/s41388-019-0919-y (2019).

17 Azuma, T. et al. B7-H1 is a ubiquitous antiapoptotic receptor on cancer cells. Blood 111, 3635-3643, doi:10.1182/blood-2007-11-123141 (2008).

18 Escors, D. et al. The intracellular signalosome of PD-L1 in cancer cells. Signal Transduct Target Ther 3, 26, doi:10.1038/s41392-018-0022-9 (2018).

19 Mastri, M. et al. Tumor-Independent Host Secretomes Induced By Angiogenesis and Immune-Checkpoint Inhibitors. Mol Cancer Ther 17, 1602-1612, doi:10.1158/15357163.MCT-17-1066 (2018).

20 Benci, J. L. et al. Tumor Interferon Signaling Regulates a Multigenic Resistance Program to Immune Checkpoint Blockade. Cell 167, 1540-1554 e1512, doi:10.1016/j.cell.2016.11.022 (2016).

21 Benci, J. L. et al. Opposing Functions of Interferon Coordinate Adaptive and Innate Immune Responses to Cancer Immune Checkpoint Blockade. Cell 178, 933-948 e914, doi:10.1016/j.cell.2019.07.019 (2019).

22 Vraetz, T. et al. Regulation of beta2-microglobulin expression in different human cell lines by proinflammatory cytokines. Nephrol Dial Transplant 14, 2137-2143, doi:10.1093/ndt/14.9.2137 (1999).

23 Sade-Feldman, M. et al. Resistance to checkpoint blockade therapy through inactivation of antigen presentation. NatCommun 8, 1136, doi:10.1038/s41467-017-01062-w (2017).

24 Garcia-Diaz, A. et al. Interferon Receptor Signaling Pathways Regulating PD-L1 and PD-L2 Expression. Cell reports 19, 1189-1201, doi:10.1016/j.celrep.2017.04.031 (2017).

25 Jacquelot, N. et al. Sustained Type I interferon signaling as a mechanism of resistance to PD-1 blockade. Cell Res, doi:10.1038/s41422-019-0224-x (2019).

26 Chen, J. et al. Type I IFN protects cancer cells from CD8+ T cell-mediated cytotoxicity after radiation. J Clin Invest 129, 4224-4238, doi:10.1172/JCI127458 (2019).

27 Chen, L. et al. CD38-Mediated Immunosuppression as a Mechanism of Tumor Cell Escape from PD-1/PD-L1 Blockade. Cancer Discov 8, 1156-1175, doi:10.1158/21598290.CD-17-1033 (2018).

28 Boukhaled, G. M., Harding, S. \& Brooks, D. G. Opposing Roles of Type I Interferons in Cancer Immunity. Annu Rev Pathol 16, 167-198, doi:10.1146/annurev-pathol-031920093932 (2021).

29 Cheon, H., Borden, E. C. \& Stark, G. R. Interferons and their stimulated genes in the tumor microenvironment. Seminars in oncology 41, 156-173, doi:10.1053/j.seminoncol.2014.02.002 (2014).

30 Parker, B. S., Rautela, J. \& Hertzog, P. J. Antitumour actions of interferons: implications for cancer therapy. Nature reviews. Cancer 16, 131-144, doi:10.1038/nrc.2016.14 (2016).

31 Lan, Y. et al. Enhanced preclinical antitumor activity of M7824, a bifunctional fusion protein simultaneously targeting PD-L1 and TGF-beta. Sci Trans/ Med 10, doi:10.1126/scitranslmed.aan5488 (2018). 
32 Clift, R., Souratha, J., Garrovillo, S. A., Zimmerman, S. \& Blouw, B. Remodeling the Tumor Microenvironment Sensitizes Breast Tumors to Anti-Programmed Death-Ligand 1 Immunotherapy. Cancer research 79, 4149-4159, doi:10.1158/0008-5472.CAN-18-3060 (2019).

33 Schofield, D. J. et al. Activity of murine surrogate antibodies for durvalumab and tremelimumab lacking effector function and the ability to deplete regulatory $T$ cells in mouse models of cancer. MAbs 13, 1857100, doi:10.1080/19420862.2020.1857100 (2021).

34 Snell, L. M., McGaha, T. L. \& Brooks, D. G. Type I Interferon in Chronic Virus Infection and Cancer. Trends Immuno/38, 542-557, doi:10.1016/j.it.2017.05.005 (2017).

35 Rusinova, l. et al. Interferome v2.0: an updated database of annotated interferonregulated genes. Nucleic Acids Res 41, D1040-1046, doi:10.1093/nar/gks1215 (2013).

36 Mosely, S. I. et al. Rational Selection of Syngeneic Preclinical Tumor Models for Immunotherapeutic Drug Discovery. Cancer immunology research 5, 29-41, doi:10.1158/2326-6066.CIR-16-0114 (2017).

37 Liu, H. et al. Tumor-derived IFN triggers chronic pathway agonism and sensitivity to ADAR loss. Nat Med 25, 95-102, doi:10.1038/s41591-018-0302-5 (2019).

38 Weichselbaum, R. R. et al. An interferon-related gene signature for DNA damage resistance is a predictive marker for chemotherapy and radiation for breast cancer. Proc Natl Acad Sci U S A 105, 18490-18495, doi:10.1073/pnas.0809242105 (2008).

39 Thorsson, V. et al. The Immune Landscape of Cancer. Immunity 48, 812-830 e814, doi:10.1016/j.immuni.2018.03.023 (2018).

40 Liberzon, A. et al. The Molecular Signatures Database (MSigDB) hallmark gene set collection. Cell Syst 1, 417-425, doi:10.1016/j.cels.2015.12.004 (2015).

41 Higgs, B. W. et al. Interferon Gamma Messenger RNA Signature in Tumor Biopsies Predicts Outcomes in Patients with Non-Small Cell Lung Carcinoma or Urothelial Cancer Treated with Durvalumab. Clinical cancer research : an official journal of the American Association for Cancer Research 24, 3857-3866, doi:10.1158/1078-0432.CCR-17-3451 (2018).

42 Sceneay, J. et al. Interferon Signaling Is Diminished with Age and Is Associated with Immune Checkpoint Blockade Efficacy in Triple-Negative Breast Cancer. Cancer Discov 9, 1208-1227, doi:10.1158/2159-8290.CD-18-1454 (2019).

43 Efremova, M. et al. Targeting immune checkpoints potentiates immunoediting and changes the dynamics of tumor evolution. Nat Commun 9, 32, doi:10.1038/s41467-01702424-0 (2018).

44 Gettinger, S. et al. Impaired HLA Class I Antigen Processing and Presentation as a Mechanism of Acquired Resistance to Immune Checkpoint Inhibitors in Lung Cancer. Cancer Discov 7, 1420-1435, doi:10.1158/2159-8290.CD-17-0593 (2017).

45 Paulson, K. G. et al. Acquired cancer resistance to combination immunotherapy from transcriptional loss of class I HLA. Nat Commun 9, 3868, doi:10.1038/s41467-018-063003 (2018).

46 Schoggins, J. W. Interferon-Stimulated Genes: What Do They All Do? Annu Rev Virol 6, 567-584, doi:10.1146/annurev-virology-092818-015756 (2019). 
47 Bekisz, J., Baron, S., Balinsky, C., Morrow, A. \& Zoon, K. C. Antiproliferative Properties of Type I and Type II Interferon. Pharmaceuticals (Basel) 3, 994-1015, doi:10.3390/ph3040994 (2010).

48 Garcia-Diaz, A. et al. Interferon Receptor Signaling Pathways Regulating PD-L1 and PD-L2 Expression. Cell reports 29, 3766, doi:10.1016/j.celrep.2019.11.113 (2019).

49 Chen, Z. et al. Inference of immune cell composition on the expression profiles of mouse tissue. Sci Rep 7, 40508, doi:10.1038/srep40508 (2017).

50 Chen, Z. et al. seq-ImmuCC: Cell-Centric View of Tissue Transcriptome Measuring Cellular Compositions of Immune Microenvironment From Mouse RNA-Seq Data. Front Immuno/9, 1286, doi:10.3389/fimmu.2018.01286 (2018).

51 Smahel, M. PD-1/PD-L1 Blockade Therapy for Tumors with Downregulated MHC Class I Expression. Int J Mol Sci 18, doi:10.3390/ijms18061331 (2017).

52 Medema, J. P. et al. Blockade of the granzyme B/perforin pathway through overexpression of the serine protease inhibitor PI-9/SPI-6 constitutes a mechanism for immune escape by tumors. Proc Natl Acad Sci U S A 98, 11515-11520, doi:10.1073/pnas.201398198 (2001).

53 Bhat, P., Leggatt, G., Waterhouse, N. \& Frazer, I. H. Interferon-gamma derived from cytotoxic lymphocytes directly enhances their motility and cytotoxicity. Cell Death Dis 8 , e2836, doi:10.1038/cddis.2017.67 (2017).

54 Lindsey, W. B. et al. CD69 expression as an index of T-cell function: assay standardization, validation and use in monitoring immune recovery. Cytotherapy 9, 123132, doi:10.1080/14653240601182838 (2007).

55 Johnson, D. E., O'Keefe, R. A. \& Grandis, J. R. Targeting the IL-6/JAK/STAT3 signalling axis in cancer. Nat Rev Clin Oncol 15, 234-248, doi:10.1038/nrclinonc. 2018.8 (2018).

56 Lu, C. et al. Type I interferon suppresses tumor growth through activating the STAT3granzyme B pathway in tumor-infiltrating cytotoxic Tlymphocytes. Journalfor immunotherapy of cancer 7, 157, doi:10.1186/s40425-019-0635-8 (2019).

57 Katlinski, K. V. et al. Inactivation of Interferon Receptor Promotes the Establishment of Immune Privileged Tumor Microenvironment. Cancer Cell 31, 194-207, doi:10.1016/j.ccell.2017.01.004 (2017).

58 Schoenfeld, A. J. \& Hellmann, M. D. Acquired Resistance to Immune Checkpoint Inhibitors. Cancer cell 37, 443-455, doi:10.1016/j.ccell.2020.03.017 (2020).

59 Shah, S. et al. Clinical and molecular features of innate and acquired resistance to antiPD-1/PD-L1 therapy in lung cancer. Oncotarget 9, 4375-4384, doi:10.18632/oncotarget.23315 (2018).

60 Sharma, P., Hu-Lieskovan, S., Wargo, J. A. \& Ribas, A. Primary, Adaptive, and Acquired Resistance to Cancer Immunotherapy. Cell 168, 707-723, doi:10.1016/j.cell.2017.01.017 (2017).

61 Kalbasi, A. \& Ribas, A. Tumour-intrinsic resistance to immune checkpoint blockade. Nature reviews. Immunology 20, 25-39, doi:10.1038/s41577-019-0218-4 (2020).

62 Chocarro de Erauso, L. et al. Resistance to PD-L1/PD-1 Blockade Immunotherapy. A Tumor-Intrinsic or Tumor-Extrinsic Phenomenon? Front Pharmacol 11, 441, doi:10.3389/fphar.2020.00441 (2020). 
63 Gubin, M. M. et al. Checkpoint blockade cancer immunotherapy targets tumour-specific mutant antigens. Nature 515, 577-581, doi:10.1038/nature13988 (2014).

64 Wei, S. C. et al. Distinct Cellular Mechanisms Underlie Anti-CTLA-4 and Anti-PD-1 Checkpoint Blockade. Cell 170, 1120-1133 e1117, doi:10.1016/j.cell.2017.07.024 (2017).

65 Gubin, M. M. et al. High-Dimensional Analysis Delineates Myeloid and Lymphoid Compartment Remodeling during Successful Immune-Checkpoint Cancer Therapy. Cell 175, 1014-1030 e1019, doi:10.1016/j.cell.2018.09.030 (2018).

66 Ghebeh, H. et al. Doxorubicin downregulates cell surface B7-H1 expression and upregulates its nuclear expression in breast cancer cells: role of $\mathrm{B} 7-\mathrm{H} 1$ as an antiapoptotic molecule. Breast cancer research : BCR 12, R48, doi:10.1186/bcr2605 (2010). Gupta, H. B. et al. Tumor cell-intrinsic PD-L1 promotes tumor-initiating cell generation and functions in melanoma and ovarian cancer. Signal Transduct Target Ther 1, 16030, doi:10.1038/sigtrans.2016.30 (2016).

68 Al Zaid Siddiquee, K. \& Turkson, J. STAT3 as a target for inducing apoptosis in solid and hematological tumors. Cell Res 18, 254-267, doi:10.1038/cr.2008.18 (2008).

69 Jiang, L. et al. Direct Tumor Killing and Immunotherapy through Anti-SerpinB9 Therapy. Cell 183, 1219-1233 e1218, doi:10.1016/j.cell.2020.10.045 (2020).

70 Lee, H. T. et al. Molecular mechanism of PD-1/PD-L1 blockade via anti-PD-L1 antibodies atezolizumab and durvalumab. Sci Rep 7, 5532, doi:10.1038/s41598-017-06002-8 (2017).

71 von Pawel, J. et al. Long-term survival in patients with advanced non-small-cell lung cancer treated with atezolizumab versus docetaxel: Results from the randomised phase III OAK study. European journal of cancer 107, 124-132, doi:10.1016/j.ejca.2018.11.020 (2019).

72 Jansen, Y. J. L. et al. Discontinuation of anti-PD-1 antibody therapy in the absence of disease progression or treatment limiting toxicity: clinical outcomes in advanced melanoma. Ann Oncol 30, 1154-1161, doi:10.1093/annonc/mdz110 (2019).

73 Pokorny, R. et al. (American Society of Clinical Oncology, 2020).

74 Marron, T. U. et al. Considerations for treatment duration in responders to immune checkpoint inhibitors. Journalfor immunotherapy of cancer 9, doi:10.1136/jitc-2020001901 (2021).

75 Danson, S. et al. Are we over-treating with checkpoint inhibitors? British journal of cancer 121, 629-630, doi:10.1038/s41416-019-0570-y (2019).

76 Chen, P. L. et al. Analysis of Immune Signatures in Longitudinal Tumor Samples Yields Insight into Biomarkers of Response and Mechanisms of Resistance to Immune Checkpoint Blockade. Cancer Discov 6, 827-837, doi:10.1158/2159-8290.CD-15-1545 (2016).

77 Yamazaki, N. et al. Cytokine biomarkers to predict antitumor responses to nivolumab suggested in a phase 2 study for advanced melanoma. Cancer Sci 108, 1022-1031, doi:10.1111/cas.13226 (2017).

78 Murakami, N., Borges, T. J., Yamashita, M. \& Riella, L. V. Severe acute interstitial nephritis after combination immune-checkpoint inhibitor therapy for metastatic melanoma. Clin Kidney J 9, 411-417, doi:10.1093/ckj/sfw024 (2016). 
79 Okiyama, N. \& Tanaka, R. Varied immuno-related adverse events induced by immunecheck point inhibitors - Nivolumab-associated psoriasiform dermatitis related with increased serum level of interleukin-6. Nihon Rinsho Meneki Gakkai Kaishi 40, 95-101, doi:10.2177/jsci.40.95 (2017).

80 Nixon, A. B. et al. Peripheral immune-based biomarkers in cancer immunotherapy: can we realize their predictive potential? Journal for immunotherapy of cancer 7, 325, doi:10.1186/s40425-019-0799-2 (2019).

81 Keegan, A. et al. Plasma IL-6 changes correlate to PD-1 inhibitor responses in NSCLC. Journal for immunotherapy of cancer 8, doi:10.1136/jitc-2020-000678 (2020).

82 Slaney, C. Y., Kershaw, M. H. \& Darcy, P. K. Trafficking of T cells into tumors. Cancer research 74, 7168-7174, doi:10.1158/0008-5472.CAN-14-2458 (2014).

83 Mikucki, M. E. et al. Non-redundant requirement for CXCR3 signalling during tumoricidal T-cell trafficking across tumour vascular checkpoints. Nat Commun 6, 7458, doi:10.1038/ncomms8458 (2015).

$84 \mathrm{Qu}, \mathrm{Y}$. et al. Baseline Frequency of Inflammatory Cxcl9-Expressing Tumor-Associated Macrophages Predicts Response to Avelumab Treatment. Cell reports 32, 108115, doi:10.1016/j.celrep.2020.108115 (2020).

85 Lunardi, S., Lim, S. Y., Muschel, R. J. \& Brunner, T. B. IP-10/CXCL10 attracts regulatory T cells: Implication for pancreatic cancer. Oncoimmunology 4, e1027473, doi:10.1080/2162402X.2015.1027473 (2015).

86 Li, C. X. et al. CXCL10/CXCR3 signaling mobilized-regulatory T cells promote liver tumor recurrence after transplantation. J Hepatol 65, 944-952, doi:10.1016/j.jhep.2016.05.032 (2016).

87 Wang, Z. et al. Peyer's patches-derived $C D 11 b(+)$ B cells recruit regulatory $T$ cells through CXCL9 in dextran sulphate sodium-induced colitis. Immunology 155, 356-366, doi:10.1111/imm.12977 (2018).

88 Ramos-Casals, M. et al. Immune-related adverse events of checkpoint inhibitors. Nat Rev Dis Primers 6, 38, doi:10.1038/s41572-020-0160-6 (2020).

89 Rotz, S. J. et al. Severe cytokine release syndrome in a patient receiving PD-1-directed therapy. Pediatr Blood Cancer 64, doi:10.1002/pbc.26642 (2017).

90 Ceschi, A., Noseda, R., Palin, K. \& Verhamme, K. Immune Checkpoint Inhibitor-Related Cytokine Release Syndrome: Analysis of WHO Global Pharmacovigilance Database. Front Pharmacol 11, 557, doi:10.3389/fphar.2020.00557 (2020).

91 Chheda, Z. S., Sharma, R. K., Jala, V. R., Luster, A. D. \& Haribabu, B. Chemoattractant Receptors BLT1 and CXCR3 Regulate Antitumor Immunity by Facilitating CD8+ T Cell Migration into Tumors. J Immuno/197, 2016-2026, doi:10.4049/jimmunol.1502376 (2016).

92 Zaretsky, J. M. et al. Mutations Associated with Acquired Resistance to PD-1 Blockade in Melanoma. N Engl J Med 375, 819-829, doi:10.1056/NEJMoa1604958 (2016).

93 Manguso, R. T. et al. In vivo CRISPR screening identifies Ptpn2 as a cancer immunotherapy target. Nature 547, 413-418, doi:10.1038/nature23270 (2017).

94 Torrejon, D. Y. et al. Overcoming Genetically Based Resistance Mechanisms to PD-1 Blockade. Cancer Discov 10, 1140-1157, doi:10.1158/2159-8290.CD-19-1409 (2020). 
95 Pai, C. S. et al. Clonal Deletion of Tumor-Specific T Cells by Interferon-gamma Confers Therapeutic Resistance to Combination Immune Checkpoint Blockade. Immunity 50, 477-492 e478, doi:10.1016/j.immuni.2019.01.006 (2019).

96 Lederle, W. et al. IL-6 promotes malignant growth of skin SCCs by regulating a network of autocrine and paracrine cytokines. International journalof cancer 128, 2803-2814, doi:10.1002/ijc.25621 (2011).

97 Kitamura, H. et al. IL-6-STAT3 controls intracellular MHC class II alphabeta dimer level through cathepsin S activity in dendritic cells. Immunity 23, 491-502, doi:10.1016/j.immuni.2005.09.010 (2005).

$98 \mathrm{Fu}, \mathrm{X}$. L. et al. Interleukin 6 induces M2 macrophage differentiation by STAT3 activation that correlates with gastric cancer progression. Cancer immunology, immunotherapy: Cll 66, 1597-1608, doi:10.1007/s00262-017-2052-5 (2017).

99 Tsukamoto, H., Senju, S., Matsumura, K., Swain, S. L. \& Nishimura, Y. IL-6-mediated environmental conditioning of defective Th1 differentiation dampens antitumour immune responses in old age. Nat Commun 6, 6702, doi:10.1038/ncomms7702 (2015).

100 Tsukamoto, H. et al. Immune-suppressive effects of interleukin-6 on T-cell-mediated anti-tumor immunity. Cancer Sci 109, 523-530, doi:10.1111/cas.13433 (2018).

101 Stroud, C. R. et al. Tocilizumab for the management of immune mediated adverse events secondary to PD-1 blockade. J Oncol Pharm Pract 25, 551-557, doi:10.1177/1078155217745144 (2019).

102 Doms, J., Prior, J. O., Peters, S. \& Obeid, M. Tocilizumab for refractory severe immune checkpoint inhibitor-associated myocarditis. Ann Oncol 31, 1273-1275, doi:10.1016/j.annonc.2020.05.005 (2020).

103 Flores-Toro, J. A. et al. CCR2 inhibition reduces tumor myeloid cells and unmasks a checkpoint inhibitor effect to slow progression of resistant murine gliomas. Proc Natl Acad Sci U S A 117, 1129-1138, doi:10.1073/pnas.1910856117 (2020).

104 Wang, X. et al. PD-L1 is a direct target of cancer-FOXP3 in pancreatic ductal adenocarcinoma (PDAC), and combined immunotherapy with antibodies against PD-L1 and CCL5 is effective in the treatment of PDAC. Signal Transduct Target Ther 5, 38, doi:10.1038/s41392-020-0144-8 (2020).

105 Tsushima, F. et al. Preferential contribution of B7-H1 to programmed death-1-mediated regulation of hapten-specific allergic inflammatory responses. Eur J Immuno/33, 27732782, doi:10.1002/eji.200324084 (2003).

106 Dolan, M. et al. Enhanced efficacy of sitravatinib in metastatic models of antiangiogenic therapy resistance. PloS one 14, e0220101, doi:10.1371/journal.pone.0220101 (2019).

107 Dobin, A. et al. STAR: ultrafast universal RNA-seq aligner. Bioinformatics 29, 15-21, doi:10.1093/bioinformatics/bts635 (2013).

108 Wang, L., Wang, S. \& Li, W. RSeQC: quality control of RNA-seq experiments. Bioinformatics 28, 2184-2185, doi:10.1093/bioinformatics/bts356 (2012).

109 Li, B. \& Dewey, C. N. RSEM: accurate transcript quantification from RNA-Seq data with or without a reference genome. BMC Bioinformatics 12, 323, doi:10.1186/1471-210512-323 (2011). 
110 Mastri, M. et al. A Transient Pseudosenescent Secretome Promotes Tumor Growth after Antiangiogenic Therapy Withdrawal. Cell reports 25, 3706-3720 e3708, doi:10.1016/j.celrep.2018.12.017 (2018).

111 Newman, A. M. et al. Robust enumeration of cell subsets from tissue expression profiles. Nat Methods 12, 453-457, doi:10.1038/nmeth.3337 (2015).

112 Amezquita, R. A. et al. Orchestrating single-cell analysis with Bioconductor. Nat Methods 17, 137-145, doi:10.1038/s41592-019-0654-x (2020).

113 McCarthy, D. J., Campbell, K. R., Lun, A. T. \& Wills, Q. F. Scater: pre-processing, quality control, normalization and visualization of single-cell RNA-seq data in R. Bioinformatics 33, 1179-1186, doi:10.1093/bioinformatics/btw777 (2017).

114 Ritchie, M. E. et al. limma powers differential expression analyses for RNA-sequencing and microarray studies. Nucleic Acids Res 43, e47, doi:10.1093/nar/gkv007 (2015). 
a

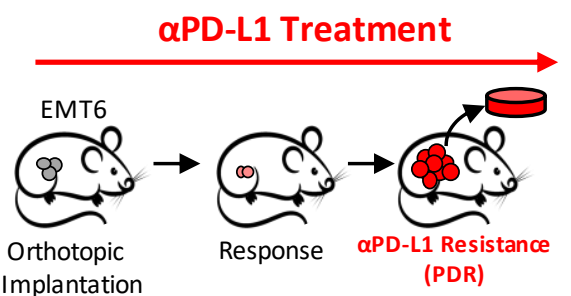

b

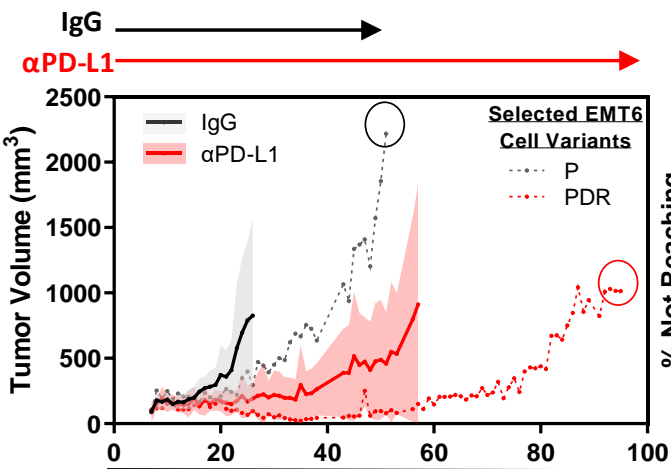

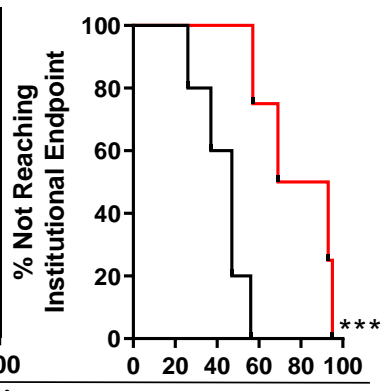

Days Post-Implantation

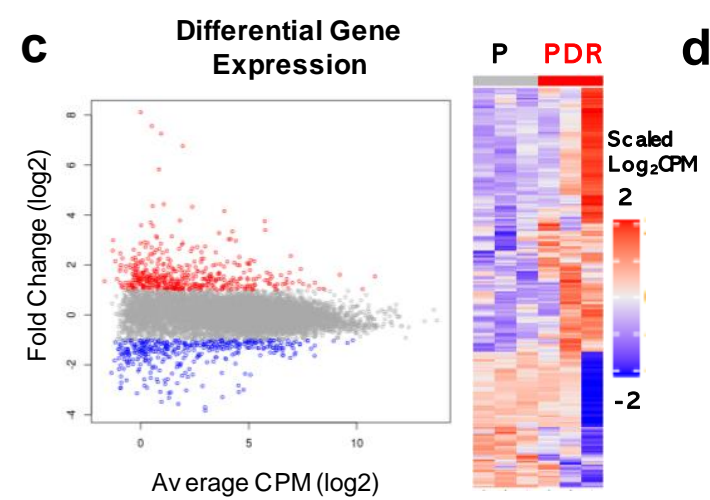

d

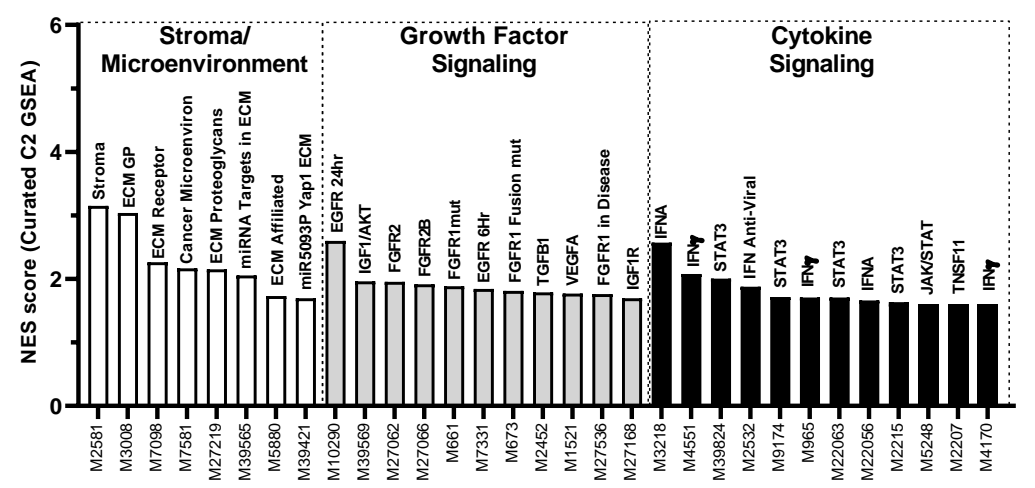

e

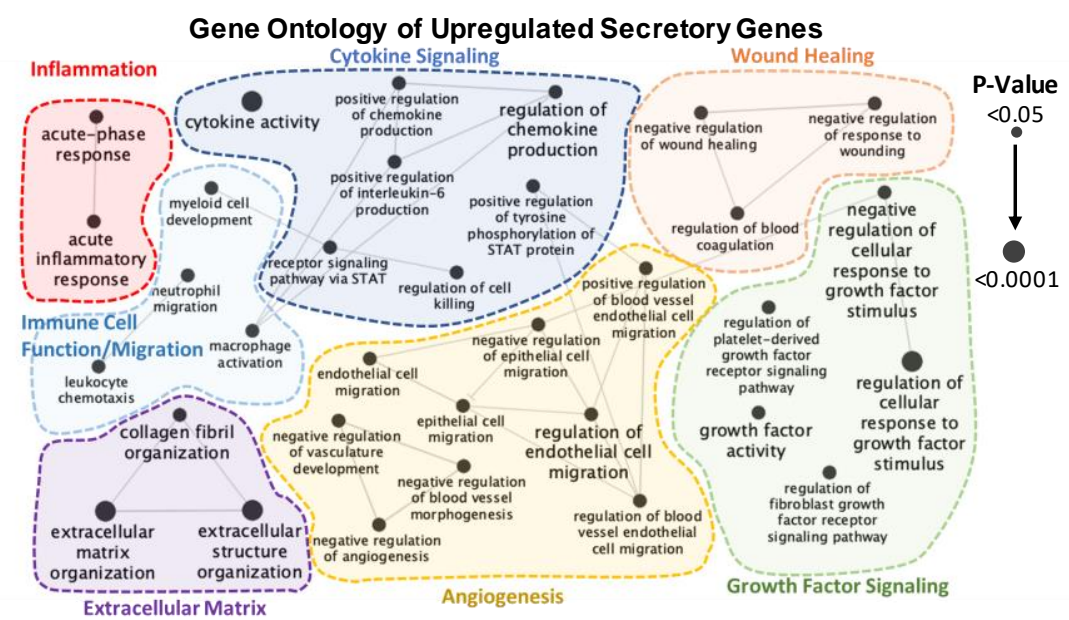

g

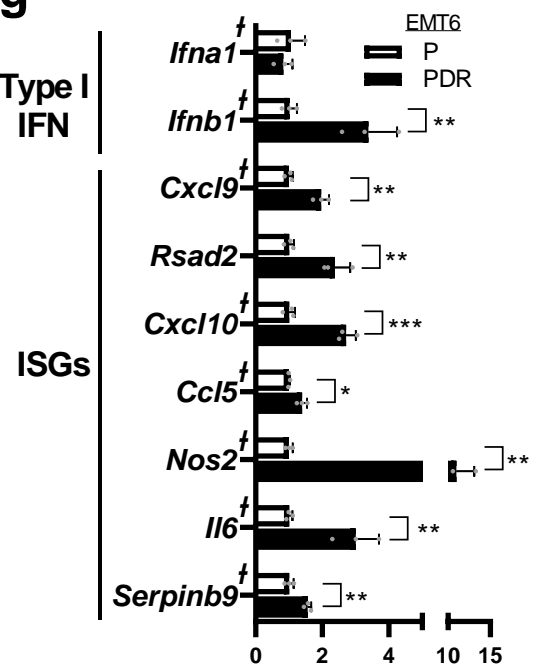

h
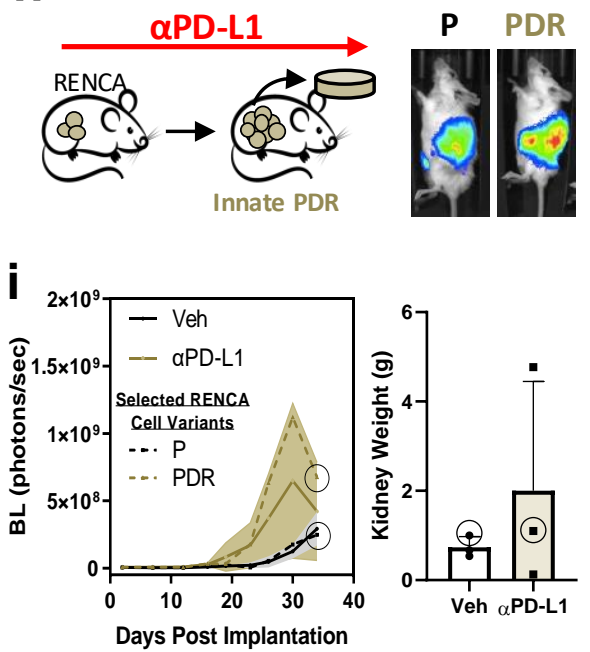

f

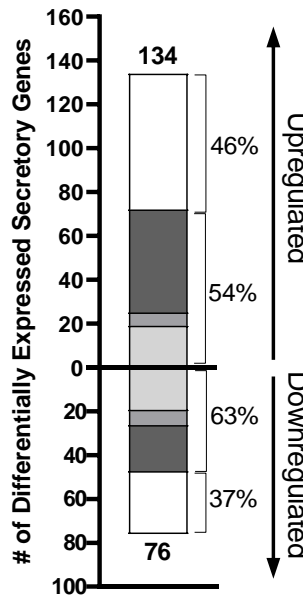

Genes Regulated By IFN Signaling (Interferome Analysis)

$\square$ None

Type I

Type II

Both

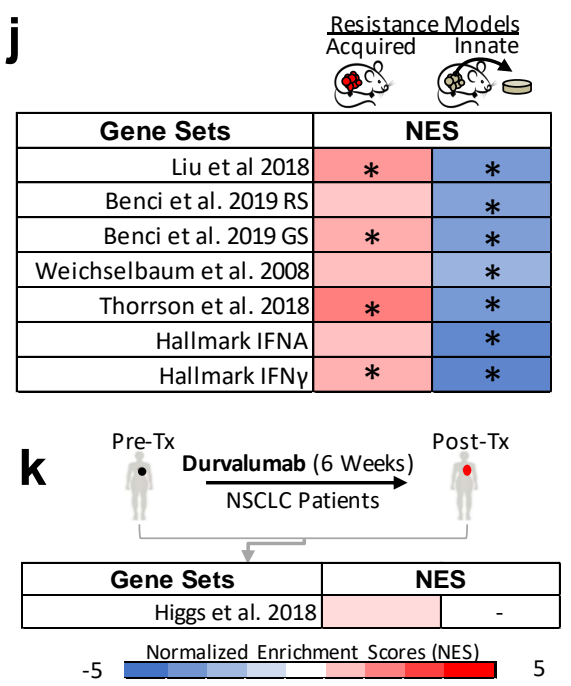




\section{Figure 1 Acquired resistance anti-PD-L1 drug resistance (PDR) increases}

secretory profiles enriched for ty pe IIFN regulated genes

(a) Schematic showing orthotopic breast EMT6 model of acquired resistance to aPD-L1 inhibition

(b) Continuous aPD-L1 treatment in Balb/c mice ( $n=3-4)$ bearing orthotopically-implanted mouse mammary EMT6 cells (left) and the time taken to reach endpoint defined by primary tumor size $\left(2000 \mathrm{~m}^{3}\right)$ and animal morbidity.

(c-f) RNA sequencing analysis of EMT6-P and EMT6-PDR tumor tissues.

c) Differentially expressed genes ( $\log _{2}$ [Fold Change] $\leq-2$ or $\geq 2$ ) in EMT6-PDR as summarized by dot plot (left) and heatmap (right). Red = upregulated; blue = downregulated.

d) Summary of stroma/tumor microenvironment, growth factor signaling, and cytokine signaling gene sets with significant positive enrichment found in PDR tumors via GSEA of all canonical pathways (C2, Molecular Signatures Database Collection).

(e) Cytoscape GO analysis of significantly enriched biological processes in upregulated secretory genes, grouped by signaling categories. Size of circles correspond to FDR significance of each process and lines represent term-term interactions defined by Kappa score.

(f) Bar graph representing Interferome Database secretory genes up- and down-regulated in EMT6-PDR (compared to EMT6-P).

(g) Type I IFNs and additional ISGs in EMT6-PDR selected cell variants (qRTPCR). + represent genes associated with secretory proteins.

(h) Schematic showing orthotopic kidney RENCA tumor model of innate resistance to aPD-L1 inhibition (left) and BLI of selected tumor variants on day of endpoint (right).

(i) BLI quantification of murine RENCA orthotopic tumor growth (left) and kidney weight at endpoint (right) with continuous $\alpha P D-L 1$ treatment (Balb/c mice; $n=3$ ).

(j-k) GSEA of EMT6-PDR and RENCA-PDR tumor cells (compared to P controls) showing heatmaps representing (j) NES of published/Hallmark IFN gene sets, and (k), NES of an IFNspecific gene-set identified in aPD-L1-treated (durvalumab) NSCLC patients (described in Ref 41). $(-)$ indicates expression below detectable thresholds. See Table S1 for details.

Parental (P); $\alpha P D-L 1$ Drug Resistant (PDR); Gene Set Enrichment Analysis (GSEA); Gene Ontology (GO); interferon stimulated genes (ISGs); Counts per million (CPM); Bioluminescence (BLI); normalized enrichment scores (NES); Non-small cell lung carcinoma (NSCLC). $\alpha P D-L 1$ (clone 80 ) and IgG were administered at $250 \mu \mathrm{g} /$ mouse every 3 days until endpoint. Primary tumor burden was assessed by caliper measurement. Time to institutional endpoint was assessed by Kaplan-Meier. PDR cell variant tumor growth shown as dotted line and time of tumor selection shown with circle for $1 B$ and 11. Selected EMT6-PDR and EMT6-P variants were maintained in vitro with respective $\alpha P D-L 1$ or IgG antibody (see Methods for details). Quantitative data shown as mean $\pm S D .{ }^{*} p<0.05,{ }^{* *} p<0.01,{ }^{* * *} p<0.001,{ }^{* * * *} p<0.0001$ 
a

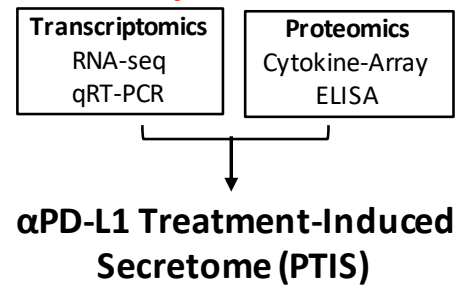

b

Sensitive

C

Insensitive
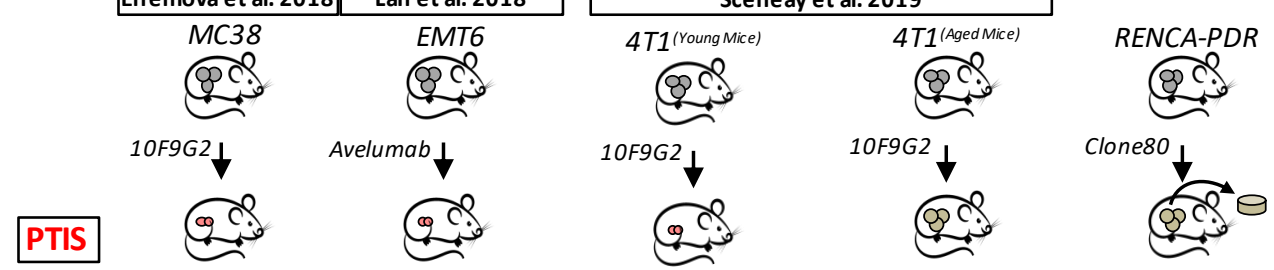

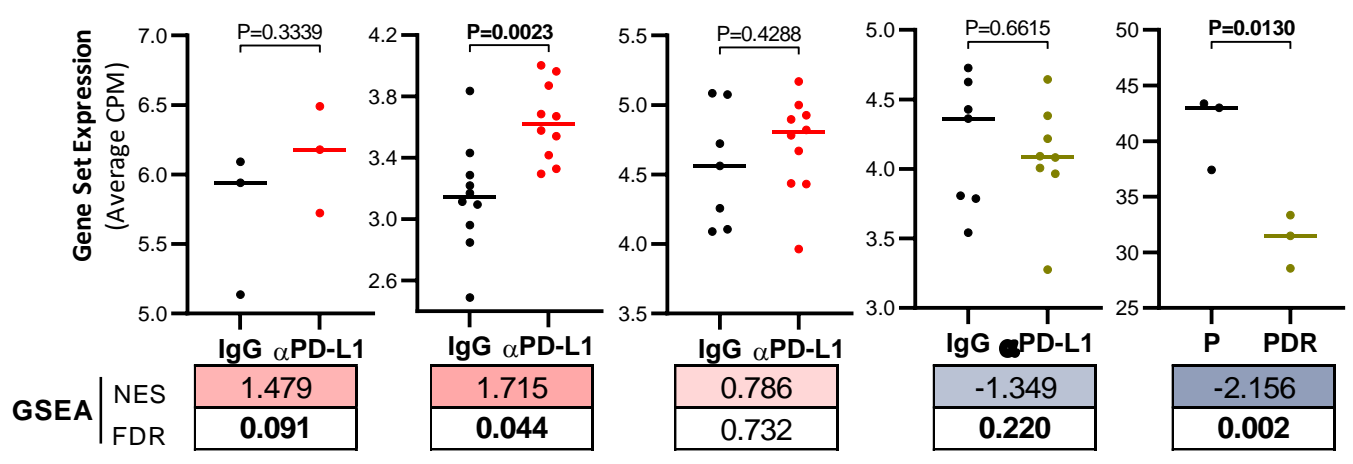

Sensitive $\rightarrow$ Acquired resistance

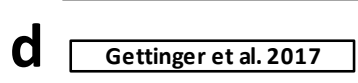

NS/S Lung Cancers

$\underset{\longrightarrow}{\alpha P D-L 1}$

PTIS

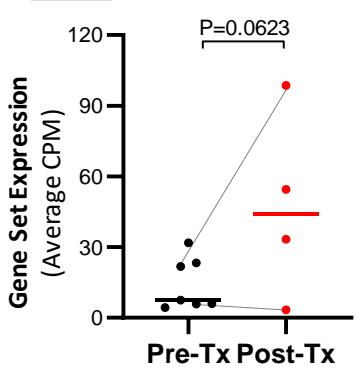

\begin{tabular}{l|l|l|} 
GSEA & $\begin{array}{l}\text { NES } \\
\text { FDR }\end{array}$ & 2.757 \\
& $<0.001$ \\
\end{tabular} e Paulson et al. 2018

Merkel Cell Carcinoma

? Avelumab $\left.\begin{array}{c}\text { MCPy } V-T \text { cell } \\ \text { Radiation }\end{array}\right]$
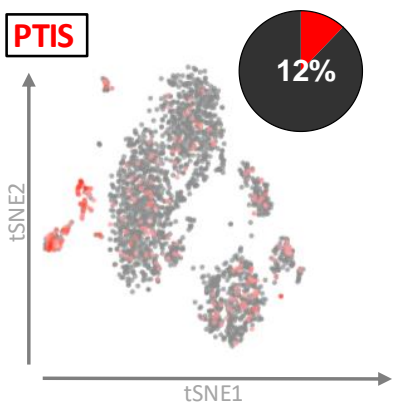

$43 \%$

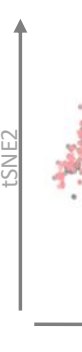

Clustered Enrichment Analysis

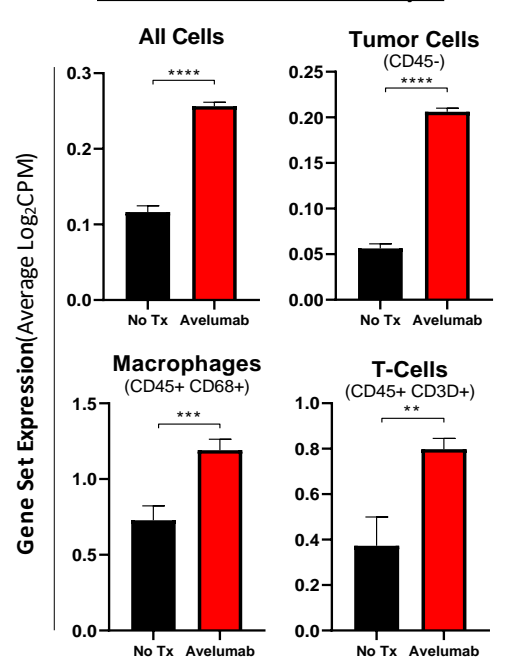




\section{Figure 2: An upregulated PTIS signature is enriched in clinical and preclinical}

models sensitive to aPD-L1 treatment

(a) Generation of an aPD-L1 treatment-induced secretome (PTIS) comprised 13 upregulated gene identified from transcriptome and proteomic analysis using EMT6-PDR cells.

(b-e) PTIS expression in published bulk and single cell RNAseq datasets involving aPD-L1 treatment in preclinical and clinical studies. RENCA-PDR model from this study included.

(b-c) Preclinical studies: PTIS expression using average CPM expression and GSEA in datasets taken from tumor models involving $\alpha P D-L 1$ treatment and found to be b) treatment-sensitive (GEO: GSE130472, GSE93017, GSE107801) or c) treatment-insensitive (GEO: GSE130472; RENCA-PDR). Data is compared to vehicle/lgG-treated controls.

(d-e) Clinical studies: PTIS expression using average CPM expression and GSEA in datasets taken from tumor biopsies of $\alpha P D-L 1$ treatment-sensitive patients.

d) NSCLC patients (dbGAP \# phs001464.v1.p1): bulk RNAseq from Pre-Tx and Post-Tx tumor sample comparisons (Gray lines indicate matched Pre- and Post-tx samples).

e) MCC patients (GEO: GSE118056): single-cell RNAseq from untreated (No-Tx) or treated (avelumab) tumor samples with Tsne plots (left) representing average $\log _{2} \mathrm{CPM}$ expression of PTIS in whole dataset, and bar graphs (right) representing clustered enrichment analysis populations identified by markers for tumors (CD45-), macrophages (CD68+), and T cells (CD3D+). Tumor sample that received No-Tx was compared to treated.

aPD-L1 Treatment-Induced Secretome (PTIS); $\alpha P D$-L1 Drug Resistant (PDR);Counts per million (CPM); Gene set enrichment analysis (GSEA); False Discovery Rate (FDR); Gene Expression Omnibus (GEO); GEO Series records (GSE); database of Genotypes and Phenotypes (dbGaP); $t$-distributed stochastic neighbor embedding (tsne); Treatment (Tx); non-small cell lung carcinoma (NSCLC); merkel cell carcinoma (MCCSignificance represented as ${ }^{*} p<0.05,{ }^{* *} p<0.01$, $p<0.001,{ }^{* * *} p<0.0001$. Bolded numbers for GSEA represent FDR $<0.25$ (see Methods). 
Fiqure 3

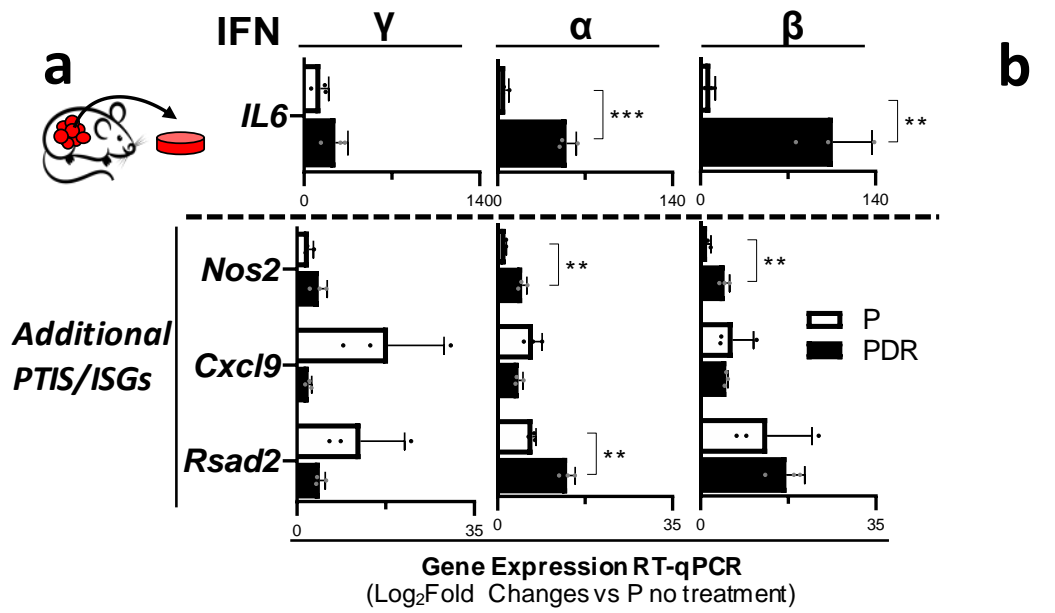

C

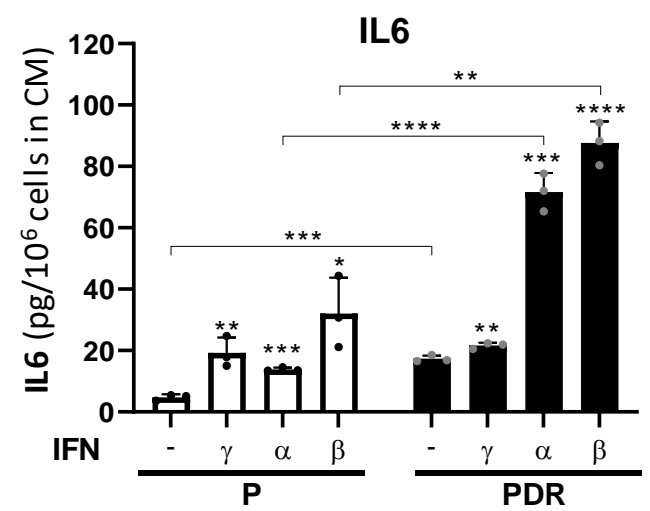

IFNAR1

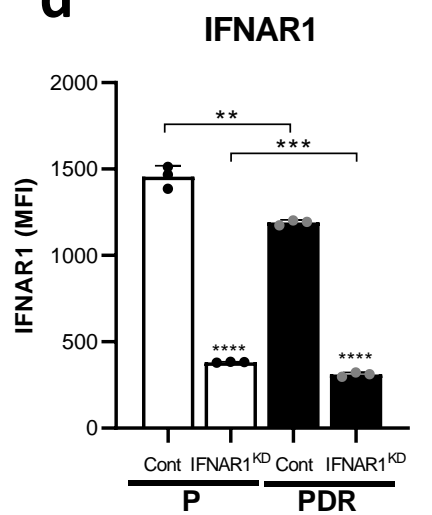

f

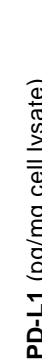

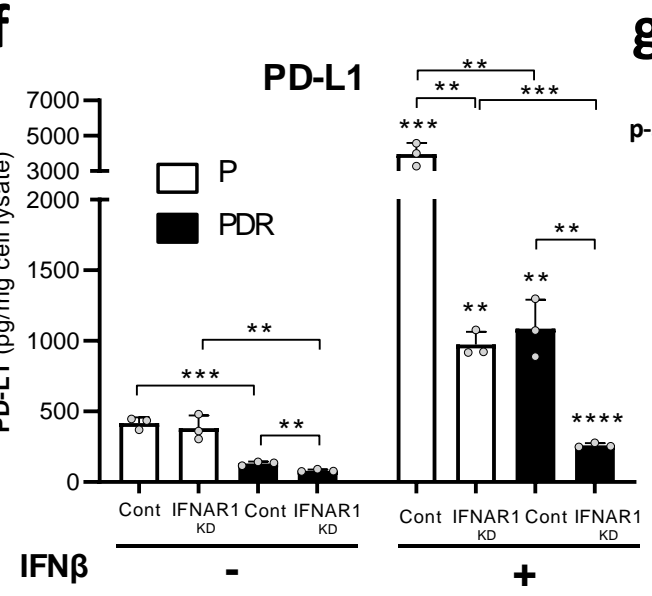

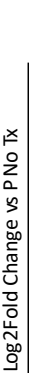

PSTAT1/STAT1 $\square$ P $\square$ PDR

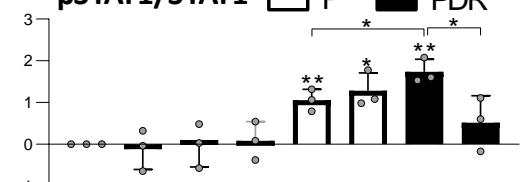

PSTAT3/STAT3 g Exposure
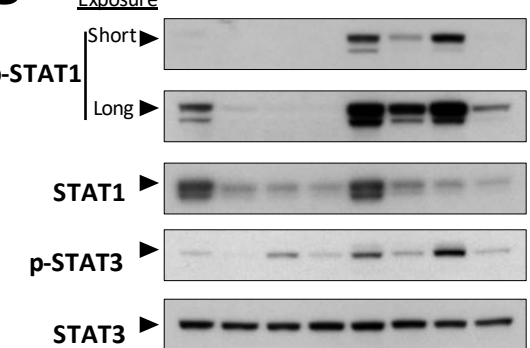

B-Actin

二- $-\frac{1}{+}+-\frac{1}{+}$ $\operatorname{IFN} \beta \frac{P}{\frac{P}{P D R}} \frac{\frac{P}{P D R}}{+}$

pSTAT3

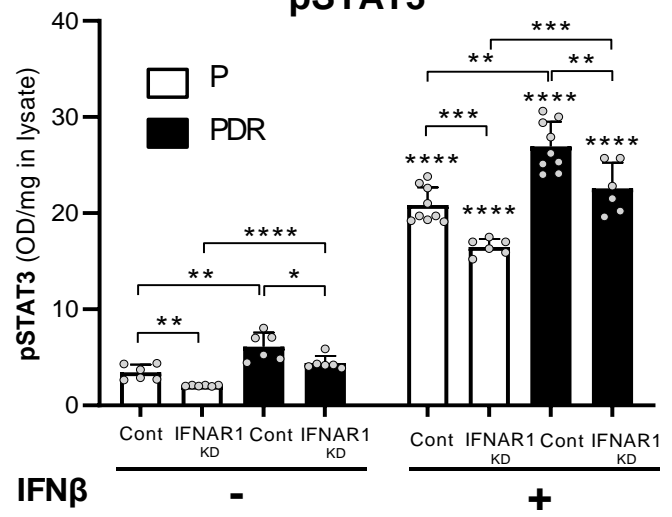

IL6

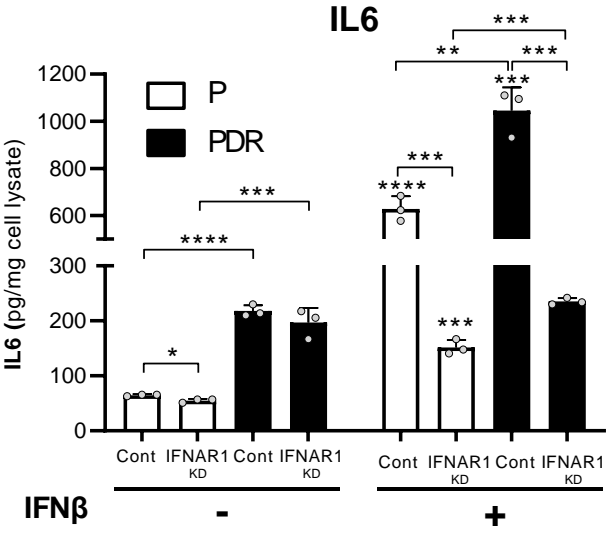

h

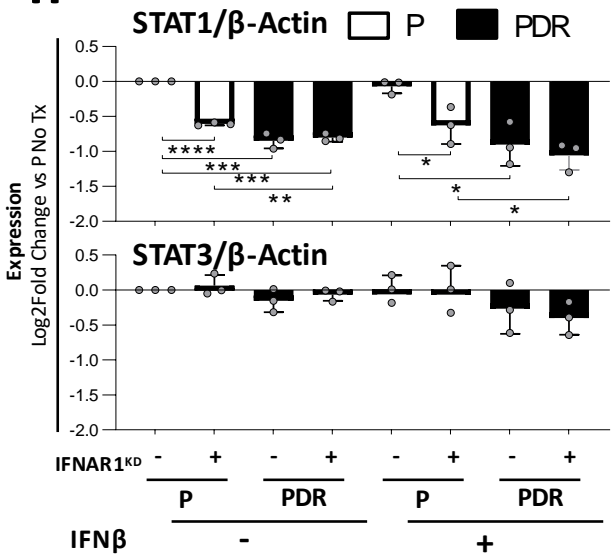

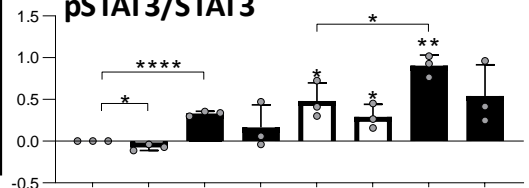

$\operatorname{IFNAR1}^{\mathrm{KO}^{-}} \frac{+}{\mathrm{P}} \frac{-}{\mathrm{PDR}} \frac{+}{\mathrm{P}} \frac{+}{\mathrm{PDR}}$ 


\section{Figure 3: Type IIFN stimulation enhances PTIS after acquired PD-L1 resistance}

(a) ISGs after stimulation with type VII IFNs in EMT6-PDR cells shown as relative to untreated $P$ controls and represented as bar graphs (qRT-PCR).

(b) heatmap summary of results in (a).

(c) Secreted IL6 levels in EMT6-P and -PDR tumor cell CM after IFNy, $\alpha$, and $\beta$ stimulation (ELISA).

(d) IFNAR1 expression in EMT6-P and -PDR before and after knockdown of IFNAR1 and respective vector controls (Flow).

(e) IL6 expression in lysates of EMT6-P and -PDR before and after knockdown of IFNAR1 ${ }^{\mathrm{KD}}$, and after IFN $\beta$ stimulation (ELISA).

(f) PD-L1 expression in lysates of EMT6-P and -PDR before and after knockdown of IFNAR1 ${ }^{\mathrm{KD}}$, and after IFN $\beta$ stimulation (ELISA).

(g) Phosphorylated and total levels of STAT $1 / 3$ in lysates of EMT6-P and -PDR before and after knockdown of IFNAR1 ${ }^{\mathrm{KD}}$ following IFN $\beta$ stimulation (Western Blot).

$(\mathbf{h}-\mathbf{i})$ Densitometry quantification of western blots shown in $(\mathrm{G})$ representing $(\mathbf{H})$ total STAT1/3 levels compared to b-actin, and (I) relative phosphorylated STAT1/3 levels compared to total STAT $1 / 3$ levels.

(j) PSTAT3 expression in lysates of EMT6-P and -PDR before and after knockdown of IFNAR1 ${ }^{\mathrm{KD}}$, and after IFNB stimulation (ELISA). (ELISA).

Parental (P); PD-L1 Drug Resistant (PDR); Conditioned Media (CM); Mean Fluorescent Intensity (MFI); IFN stimulated genes (ISGs); IFNAR1 knockdown (IFNAR1 ${ }^{K D}$ ) shRNA vector control (Cont); Cells were treated with $10 \mathrm{ng} / \mathrm{ml}$ of IFNs and collected after 15mins (pSTAT3 shown in G), 48 hours (gene expression shown in $A-B$ ), and 5 days (proteins shown in $C-F$ ). ${ }^{*} p<0.05$, ** $p<0.01,{ }^{* * *} p<0.001,{ }^{* * * *} p<0.0001$ indicate significance compared untreated controls unless otherwise shown (lines). 

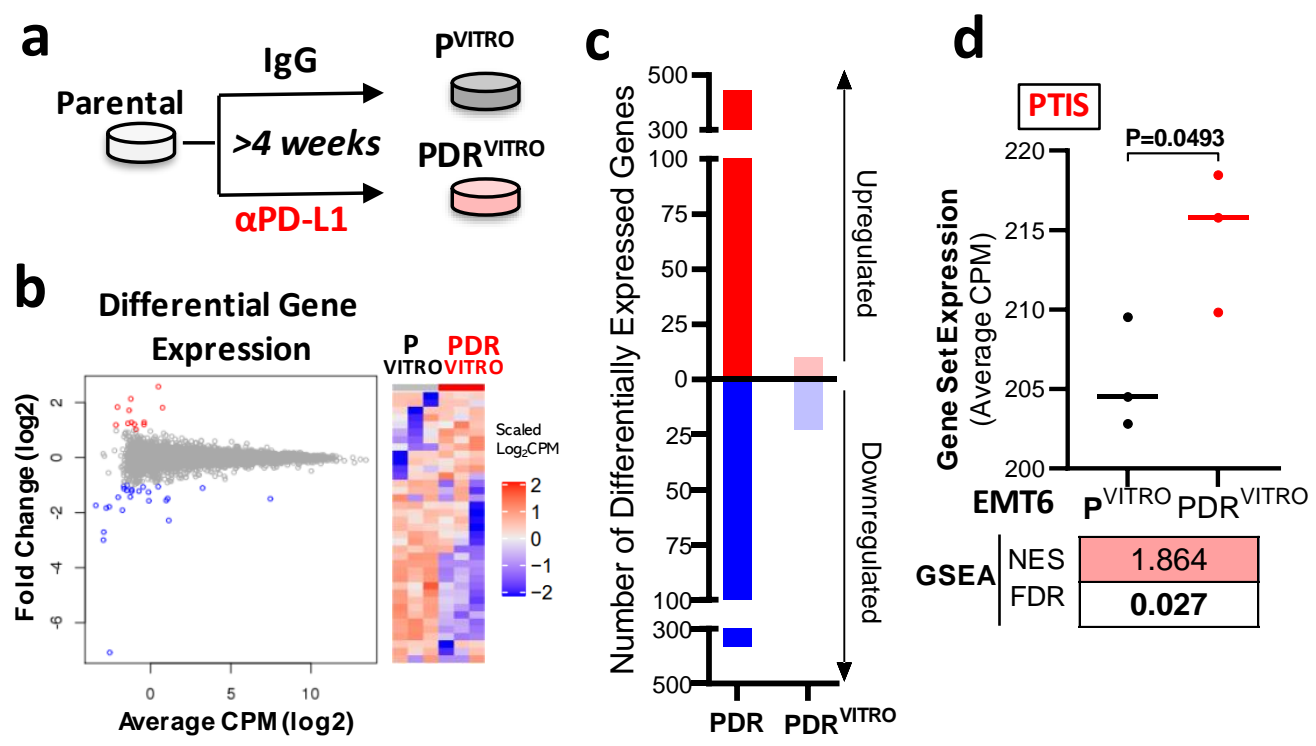

e

EMT6-PDR ${ }^{\text {VITRO }}$

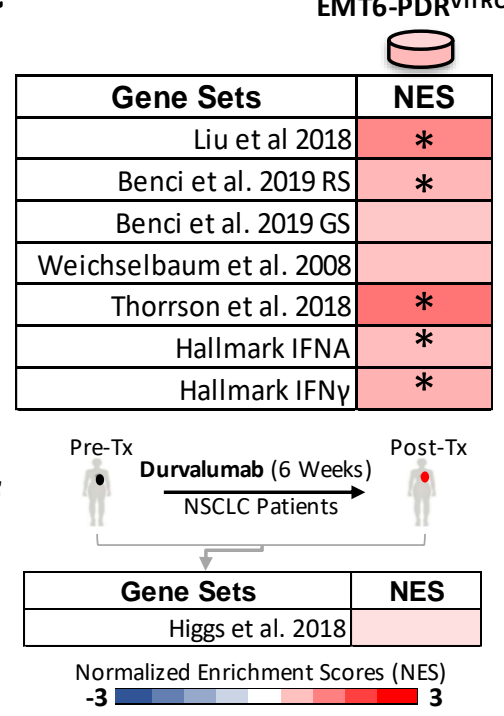

$\boldsymbol{g}^{\text {EMT6-PDR }}$

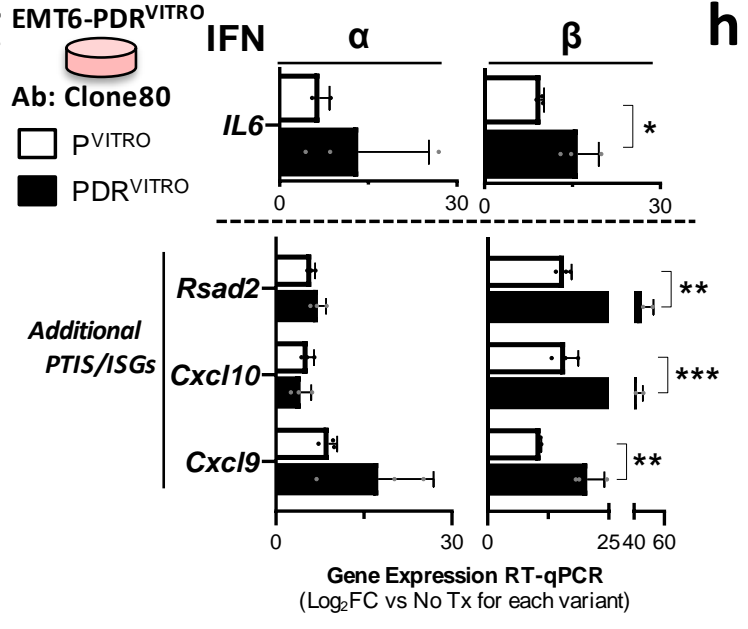

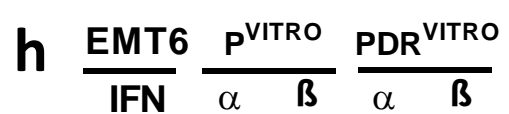

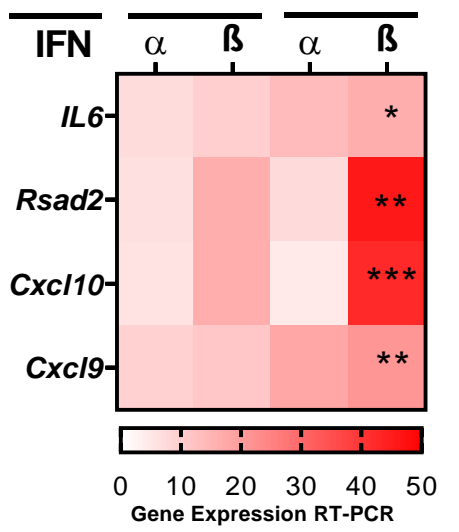

(Average Log2FC vs No Tx for each variant)

CT26-PDR VITRO

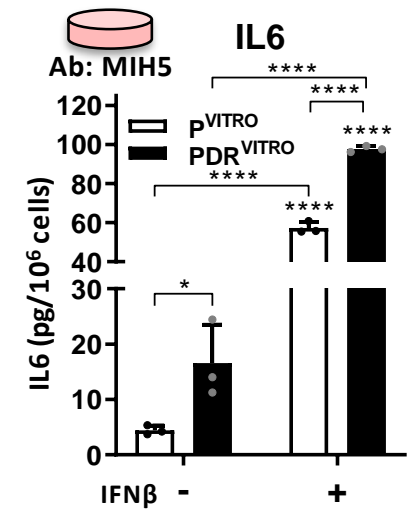

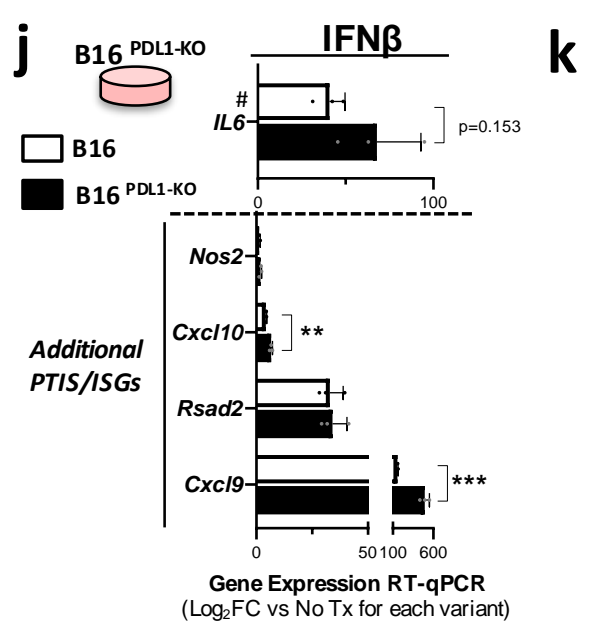

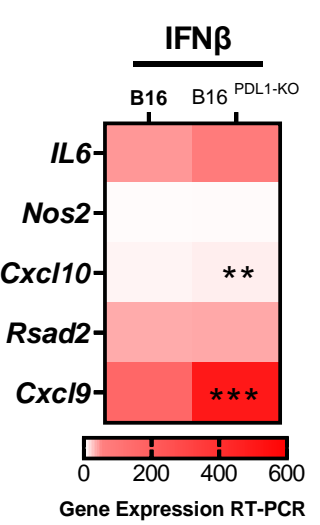

(Average Log2FC vs No Tx for each variant)
EMT6-PDR ${ }^{\text {VITRO }}$

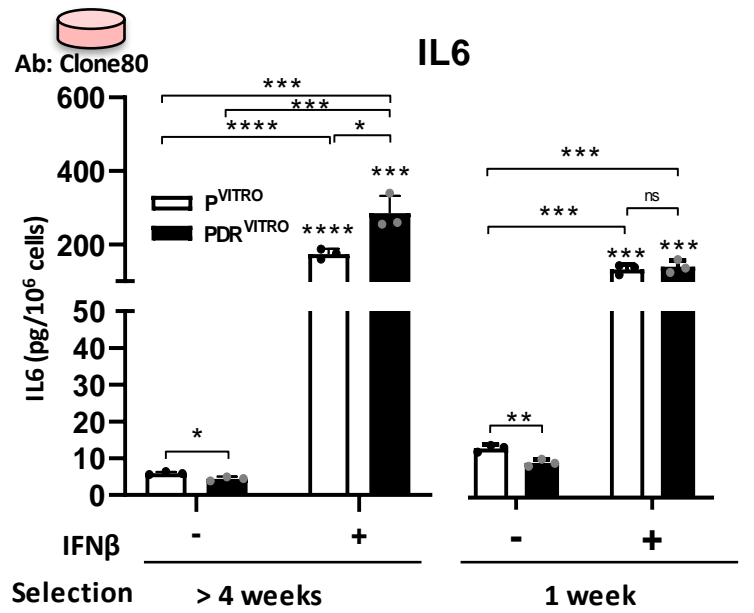




\section{Figure 4: Persistent PD-L1 blockade in vitro sensitizes tumor cells to Type I}

IFN stimulation of PTIS

(a) Schematic showing generation of PDR ${ }^{V I R O}$ cell variants following aPD-L1 treatment in vitro for $>4$ weeks.

(b-e) RNA sequencing analysis of EMT6-PVITRO and -PDR VITRO (aPD-L1: Clone80) cell variants.

(b) Differentially expressed genes ( $\log _{2}$ [Fold Change] $\leq-2$ or $\geq 2$ ) in EMT6-PDRVITRO as summarized by dot plot (left) and heatmap (right). Red = upregulated; blue = downregulated.

(c) Bar graph summarizing upregulated (red) and downregulated (blue) genes from in vivo-derived EMT6 PDR (versus P) and in vitro-derived EMT6-PDR VITRO (versus PVITRO)

(d) Average CPM expression and GSEA of PTIS in EMT6 PDR VITRO dataset.

(e-f) GSEA of EMT6-PDR ${ }^{V I T R O}$ (compared to PVITRO controls) showing heatmaps representing (E) NES of published/Hallmark IFN gene sets, and (F), NES of an IFN-specific gene-set identified in aPD-L1-treated (durvalumab) NSCLC patients (described in Ref 41). (-) indicates expression below detectable thresholds. See Table S1 for details.

(g) ISGs after stimulation with type I IFNs in EMT6-PDR VITRO cells shown as relative to untreated controls for each variant and represented as bar graphs (qRTPCR).

(h) heatmap summary of results in (g).

(i) IL6 expression in CM of CT26-PDR ${ }^{\text {VITRO }}$ ( $\alpha$ PD-L1: MIH5) and P control cell variants after IFN $\beta$ stimulation (ELISA).

(j) ISGs after stimulation with IFN $\beta$ in $\mathrm{B} 16^{\mathrm{PDL} 1-\mathrm{KO}}$ cells shown as relative to untreated controls for each variant and represented as bar graphs (qRTPCR). \# indicating genes with low levels of expression where increased cDNA input was required for reaction.

(k) heatmap summary of results in (j).

(l) IL6 expression after IFN 3 stimulation in CM of EMT6-PDR VITRO and P control cell variants derived after 1 or $>4$ weeks of aPD-L1 (clone 80) treatment (ELISA).

Parental (P); $P D-L 1$ Drug Resistant (PDR); in vitro derived Parental (PVITRO); in vitro derived PD-L1 Drug Resistant (PDR VITRO); Gene Set Enrichment Analysis (GSEA); aPD-L1 Treatment-Induced Secretome (PTIS); interferon stimulated genes (ISGs); normalized enrichment scores (NES); false discovery rate (FDR); Conditioned media (CM); not significant (NS); PD-L1 knockout (PDL1-KO); complementary DNA (cDNA). Cells were treated with $10 \mathrm{ng} / \mathrm{ml}$ of IFNs and collected after 48 hours for gene expression quantification and after 5 days for IL6 protein expression quantifications. * $p<0.05,{ }^{* *} p<0.01,{ }^{* *} p<0.001,{ }^{* * *} p<0.0001$ except for GSEA where bolded numbers and (*) indicate $F D R<0.25$ 
a Total Immune Score

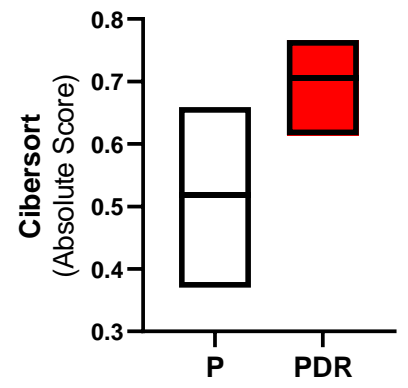

b CIBERSORT (Im mUCC)

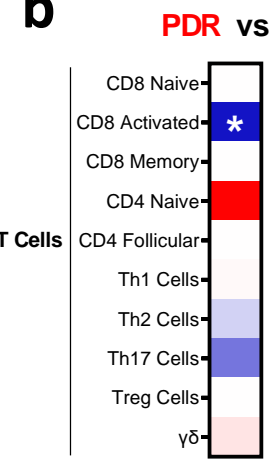

PR vs $P \quad P D R$ vs $P$

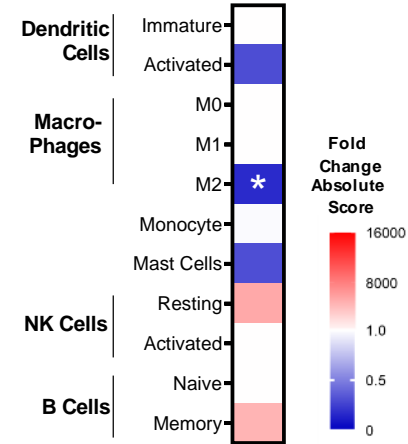

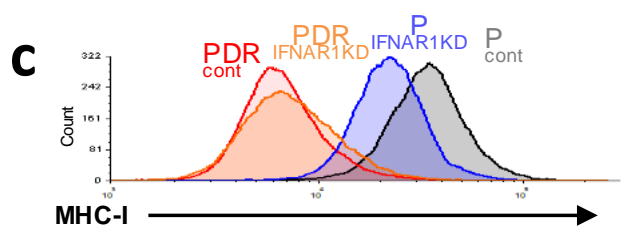
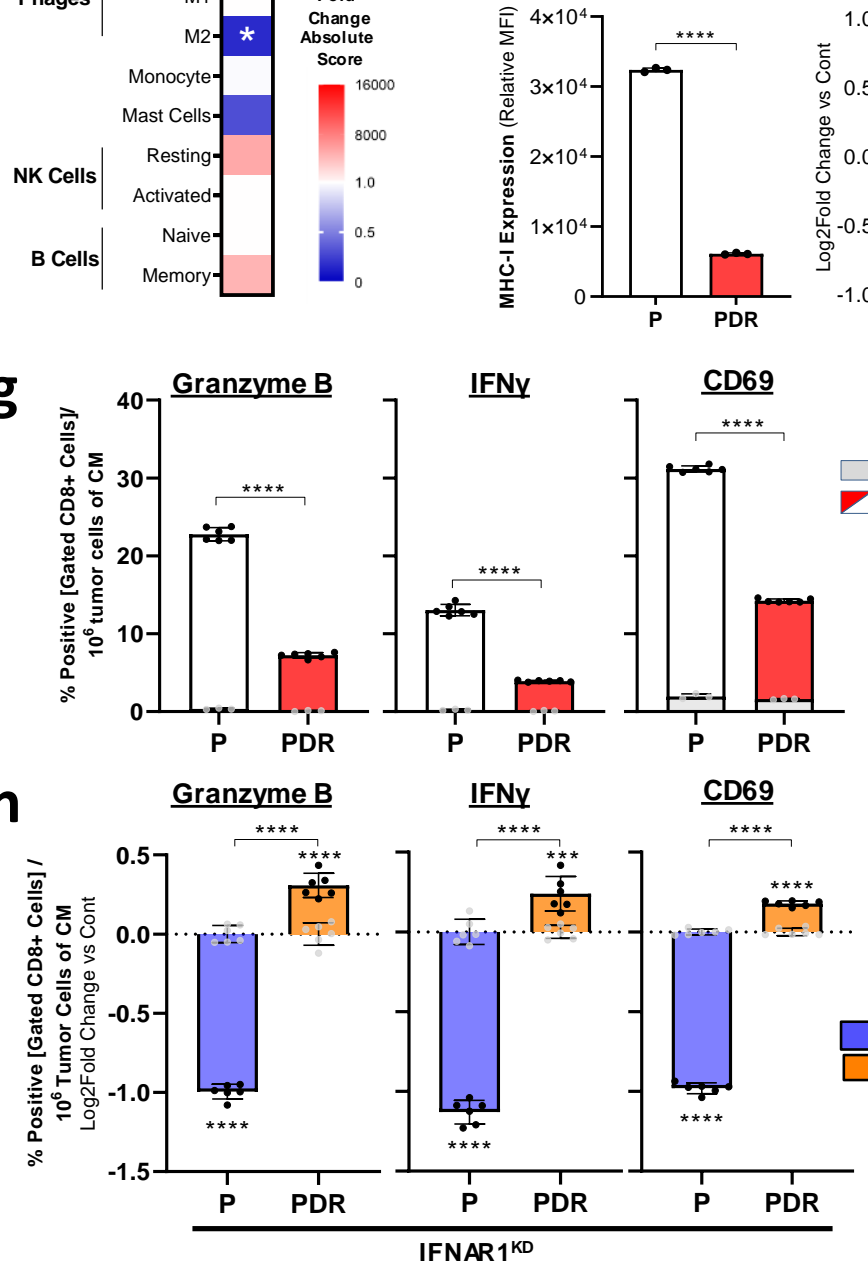

7-AAD

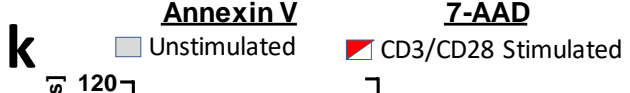

FNy

g
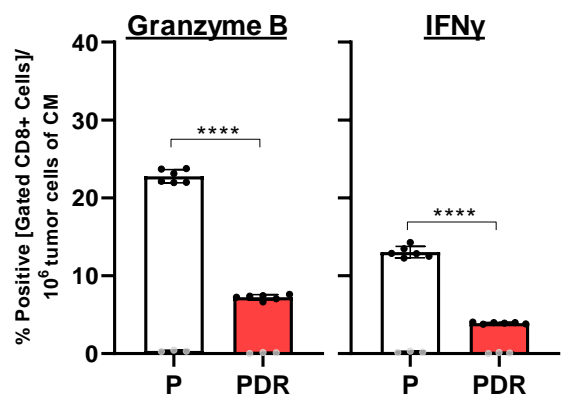

$\square$ Unstimulated

CD3/CD28 Stimulated
IFNAR1 ${ }^{\text {KD }}$ vs. P

IFNAR1 ${ }^{\mathrm{KD}}$ vs. PDR

\section{EMT6-PDR}

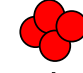

Co-culture

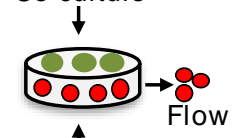

aCD3/CD28

Stimulation

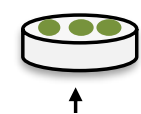

Splenocytes

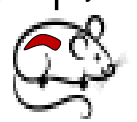

h

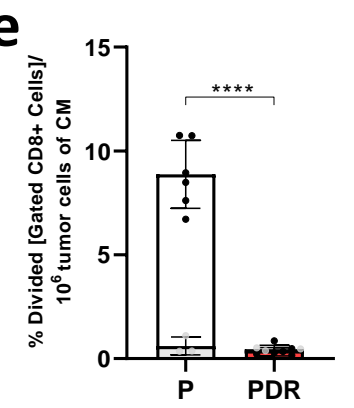

f

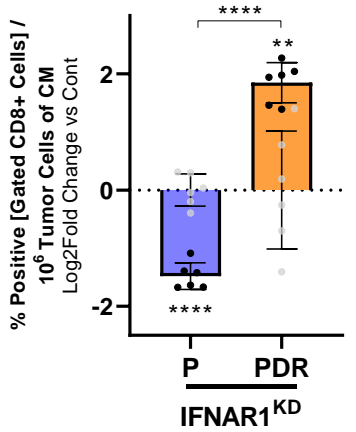

j

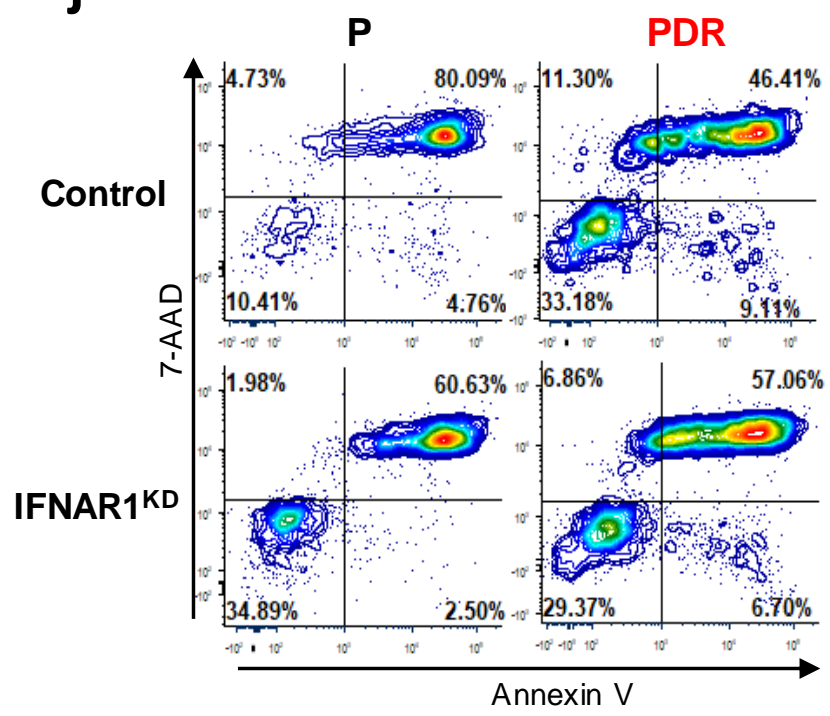

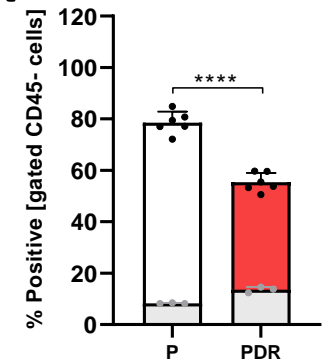

Annexin V

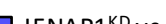

$\square$ IFNAR1 ${ }^{\mathrm{KD}}$ vs.

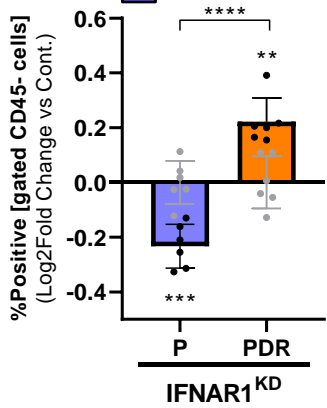

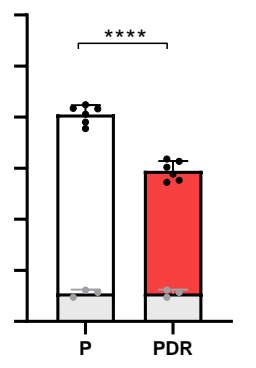

7-AAD

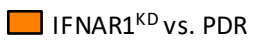

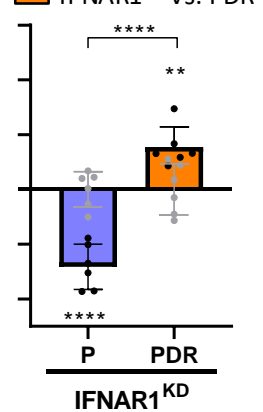




\section{Figure 5: PDR-mediated immune-protection is IFN signaling-dependent.}

(a) Cibersort tissue deconvolution analysis of EMT6-P and -PDR RNAseq data using ImmuCC mouse signature with box-plot representing absolute total immune score.

(b) Heatmap representing log2 fold change of absolute scores of various immune signatures of results from $(A)$.

(c) MHC-I H-2Kd expression in EMT6-P and -PDR cells before and after IFNAR1 knockdown shown as a histogram (top), barplot of P/PDR (bottom left), and log2-fold change comparison of EMT6-P and -PDR-IFNAR1 ${ }^{\mathrm{KD}}$ variants relative to respective vector controls (bottom right) (flow).

(d) Schematic of Balb/c-derived splenocyte proliferation and activation following incubation EMT6$P$ and -PDR CM for experiments in e-h.

(e-f) CD8+ splenocyte division (CSFE dilution) after co-incubation with (e) EMT6-P and -PDR variant $\mathrm{CM}$ and (F) EMT6-P and -PDR-IFNAR1 ${ }^{\mathrm{KD}}$ variant CM compared to controls (Flow).

(g-h) CD8+ splenocyte activation markers expression (Granzyme B, IFNy, CD69) after coincubation with (G) EMT6-P and -PDR variants and (h) EMT6-P and -PDR-IFNAR1 ${ }^{\mathrm{KD}}$ variant compared to controls (Flow).

(i) Schematic of EMT6-P and -PDR tumor cell cytotoxicity following co-culture with Balb/c-derived splenocytes for experiments in J-L (Flow).

(j-I) Apoptosis (Annexin V) and cell death (7-AAD) staining of CD45- gated tumor cells after coincubation with splenocytes showing (j) representative contour plots of stimulated splenocyte groups, (k) EMT6-P and -PDR variants, and (I) EMT6-P and -PDR-IFNAR1 ${ }^{\mathrm{KD}}$ variants compared to controls (Flow).

Parental (P); PD-L1 Drug Resistant (PDR); IFNAR1 knockdown (IFNAR1KD); Conditioned Media $(C M){ }^{*} p<0.05,{ }^{* *} p<0.01,{ }^{* * *} p<0.001,{ }^{* * * *} p<0.0001$ compared to vector controls unless noted otherwise. For $C, F, H$, and $L$ white bars represent vector controls 
Fiqure 6

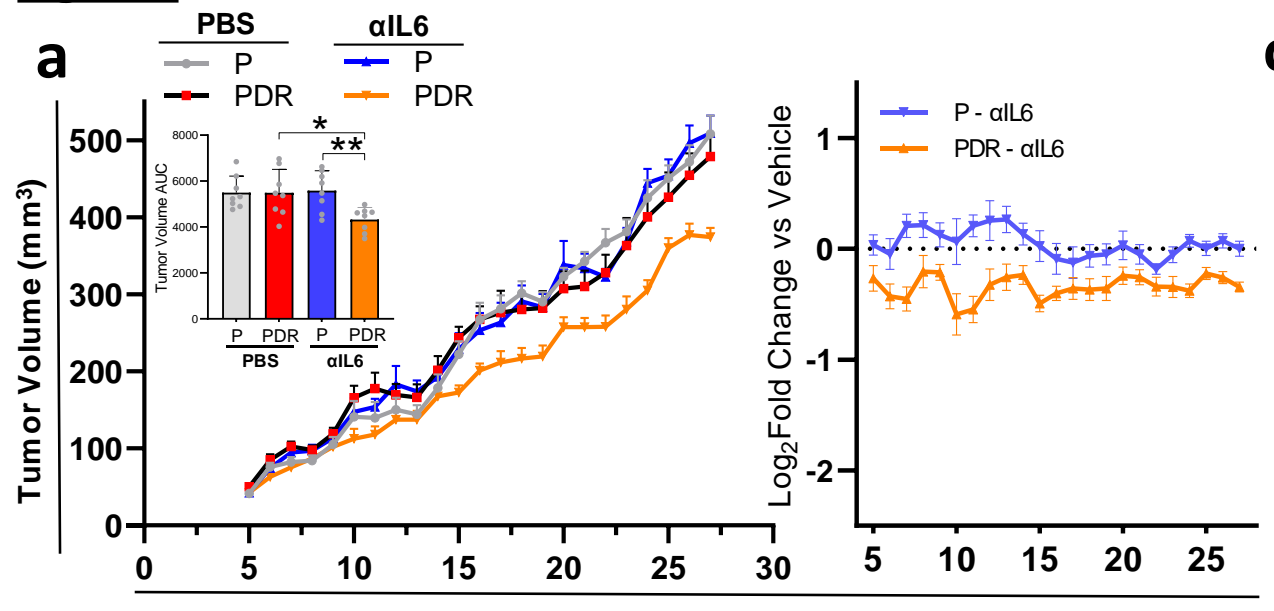

Days Post Implantation

b

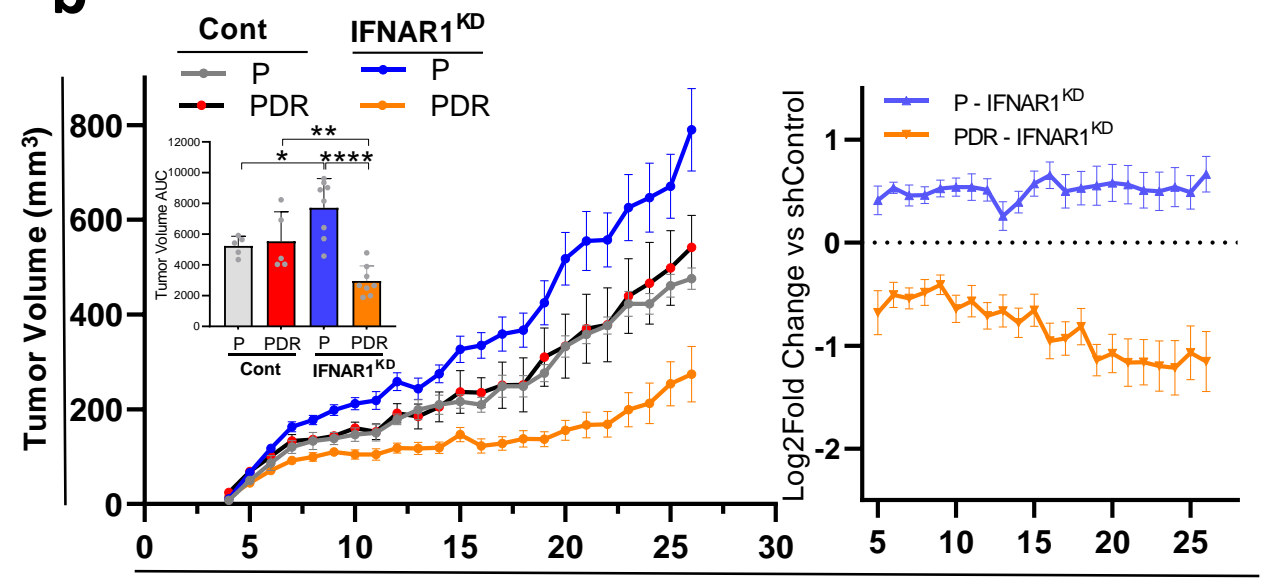

Days Post Implantation

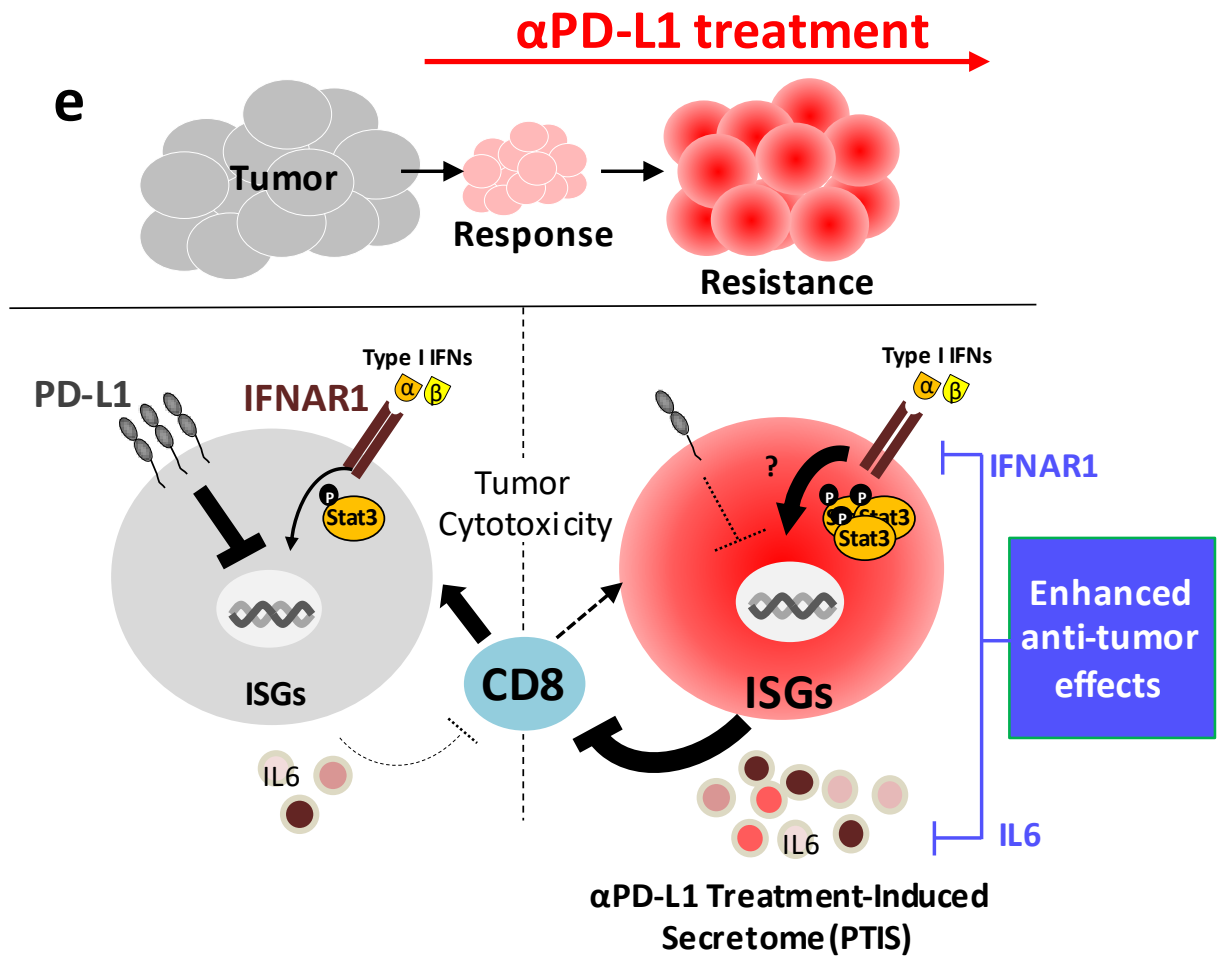

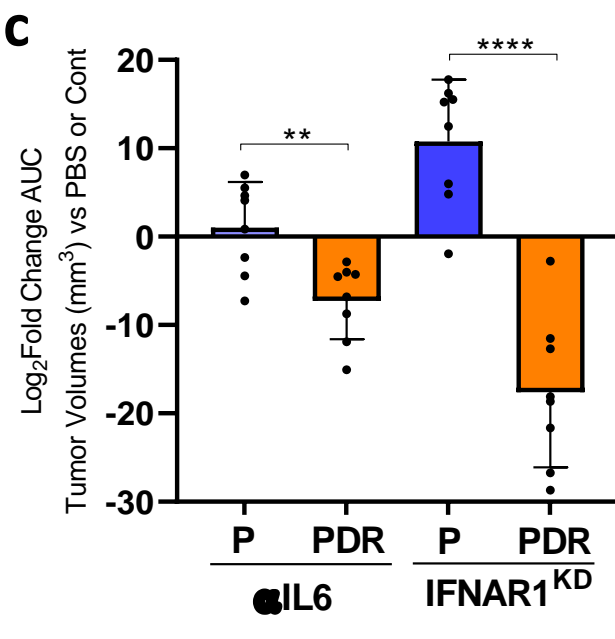

Skin/Abdominal Wall Invasion

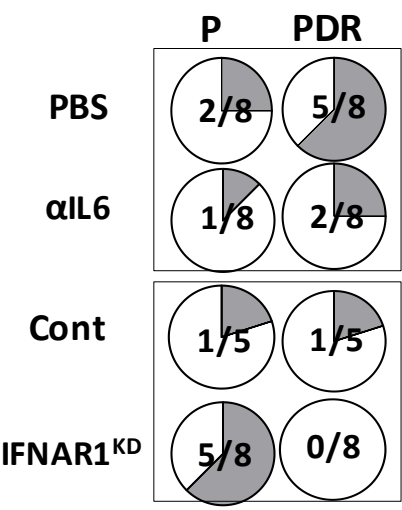




\section{Figure 6: Inhibition of PTIS regulators selectively inhibits PDR tum or growth}

(a) Orthotopic tumor growth and response to anti-IL6 upon re-implantation of EMT6-P and -PDR cell variants (left; $n=8 ; B a l b / c)$ with summary of $A U C$ analysis (left-inset). $\log _{2}$ Fold Change analysis comparing anti-IL6 to vehicle controls (right).

(b) Orthotopic tumor growth upon re-implantation of EMT6-P and -PDR -IFNAR1 ${ }^{\mathrm{KD}}$ and respective Cont cell variants (left; $n=5-8 ; B a l b / c$ ) with summary of AUC analysis (left-inset). $\log _{2}$ Fold Change analysis comparing IFNAR $1^{\mathrm{KD}}$ to respective controls (right).

(c) AUC analysis for $\log _{2}$ Fold Change of treatment or knockdown compared to vehicle or Cont, respectively, for experiments shown in a-b.

(d) Metastasis and invasion of mice bearing EMT6-P and -PDR tumors (shown in a-b) with invasion in the peritoneum wall after PBS, anti-IL6, Cont, or IFNAR1 ${ }^{K D}(n=5-8)$

(e) Proposed model of IFN-signaling 'rewired' tumor cells following acquired resistance to PD-L1 inhibition

Parental (P); PD-L1 Drug Resistant (PDR); IFNAR1 knockdown (IFNAR1 ${ }^{K D}$ ); shRNA Vector Control (Cont); Area under the curve (AUC). Anti-IL6 was administered at 100 $\mu \mathrm{g} / \mathrm{mouse} / 3$ days continuously. Primary tumor burden was assessed by caliper measurement. Quantitative data shown as mean \pm SEM. 